\title{
TLR7 in B cells promotes renal inflammation and Gd-IgA1 synthesis in IgA nephropathy
}

\author{
Nuoyan Zheng, ${ }^{1,2}$ Kaifeng Xie, ${ }^{3}$ Hongjian Ye, ${ }^{2}$ Yu Dong, ${ }^{4}$ Bing Wang, ${ }^{5}$ Ning Luo, ${ }^{2}$ Jinjin Fan, ${ }^{2}$ \\ Jiaqing Tan, ${ }^{2}$ Wei Chen, ${ }^{2}$ and Xueqing $\mathrm{Yu}^{2}$ \\ ${ }^{1}$ Translational Medical Center and ${ }^{2}$ Department of Nephrology, The First Affiliated Hospital, Sun Yat-sen University, \\ Guangzhou, China. ${ }^{3}$ Guangdong Medical University, Zhanjiang, Guangdong Province, China. ${ }^{4}$ Department of Pathology, The \\ First Affiliated Hospital, Sun Yat-sen University, Guangzhou, China. ${ }^{5}$ Guangzhou International Travel Health Care Center, \\ Guangdong Province, China.
}

TLR7 has been linked to the pathogenesis of glomerulonephritis, but its precise roles are not clear. In this study, we evaluated the roles of TLR7 in IgA nephropathy (IgAN). TLR7 proteins were abundant in CD19+ B cells infiltrated in the kidneys of patients with IgAN. The intensities of both intrarenal TLR7 and CD19 proteins were closely associated with kidney function (estimated glomerular filtration rate [eGFR] and serum creatinine concentration) and renal histopathology (tubular atrophy, leukocyte infiltration, tubulointerstitial fibrosis, and global glomerulosclerosis) in patients with IgAN. Meanwhile, TLR7 mRNA levels were significantly increased in peripheral blood $B$ cells of patients with IgAN. TLR7 ${ }^{+}$CD19+ $B$ cells expressed inflammatory cytokines (IL-6 and IL-12) in kidneys and produced high levels of IgA1 and galactose deficient-IgA1 (Gd-IgA1) in peripheral blood of patients with IgAN. Mechanistically, TLR7 activated B cells to produce high levels of GdIgA1 via the TLR7-CALNT2 axis in IgAN. Protein levels of GALNT2 were increased by overexpression of TLR7, while they were reduced by TLR7 knockdown in B cells. GALNT2 overexpression augmented Gd-IgA1 production in B cells derived from patients with IgAN. Taken together, high TLR7 expression in B cells has dual roles in the development and progression of IgAN, by facilitating renal inflammation and Gd-IgA1 antibody synthesis.

Conflict of interest: The authors have declared that no conflict of interest exists.

Copyright: (ㄷ) 2020, American Society for Clinical Investigation.

Submitted: February 3, 2020

Accepted: June 10, 2020

Published: July 23, 2020

Reference information: /CI Insight. 2020;5(14):e136965.

https://doi.org/10.1172/jici.

insight.136965.

\section{Introduction}

IgA nephropathy (IgAN) is the most common form of primary glomerulonephritis worldwide, with about $25 \%-30 \%$ patients developing end-stage renal disease within 20 years $(1,2)$. The most significant feature of IgAN is the mesangial deposition of polymeric galactose-deficient IgA1 (Gd-IgA1) immune complexes (3), which contain terminal $\beta 1,3$ galactose (Gal) deficiency and exposed $N$-acetylgalactosamine (GalNAc) residues in $O$-glycans of the hinge region of $\operatorname{IgA} 1(2,4)$. Aberrant $\mathrm{Gd}-\mathrm{IgA} 1$ antibodies produced by B cells bind to specific IgG antibodies to form immune complexes, which are difficult to remove from the circulation; the complexes are deposited on the surface of the mesangium and ultimately attack mesangial cells (5-7). In addition to acting as important pathogenic factors in IgAN, Gd-IgA1 and the Gd-IgA1 immune complexes are biomarkers of disease severity and clinical outcomes in IgAN (8-11).

Pathogen infections affecting the mucosal immune system are closely related to the pathogenesis of IgAN (12). B cells in the mucosal immune system could mature into plasma B cells in response to pathogen stimulation, resulting in IgA1 and IgA2 secretion to form dimeric IgA1, IgA2, or large polymeric IgA1 antibodies. These IgA1-producing B cells enter the circulation and migrate along the mucosa, bone marrow, and kidney, becoming tissue-resident lymphocytes (13). B cells in the peripheral blood of patients with IgAN are also able to synthesize Gd-IgA1, including both freshly isolated B cells and EBV-immortalized B cells derived from patients $(14,15)$. The synthesis of $O$-glycan of human IgA1 starts with the addition of GalNAc residues to serine or threonine residues in the hinge region of the IgA1 antibody, followed by addition of Gal residues onto GalNAc and, finally, with sialic acid residues (16). The first step in the transfer of GalNAc residues to IgA1 is mainly mediated by polypeptide $N$-acetylgalactosaminyltransferase 2, known as GALNT2 (17). The addition of Gal is mediated by core $\beta 1,3$-galactosyltransferase (C1GALT1) via its chaperone, COSMC $(18,19)$. 
The expression and activity of C1GALT1 and COSMC are important for the $O$-glycosylation of IgA1 and regulated by cytokines (e.g., IL-6 and A proliferation inducing ligand [APRIL]), and other factors (20-23).

Besides the attack of Gd-IgA1 on mesangial cells, infiltration and activation of renal immune cells also contribute to progressive kidney injury and the development of chronic kidney disease (CKD), eventually leading to renal failure (24-26). Infiltrating T cells and B cells are capable of in situ proliferation, even forming a tertiary lymphoid structure with follicular DCs $(24,27)$. Tissue-infiltrated B cells secrete proinflammatory cytokines, chemokines, and immunoglobulins, which further exaggerate renal inflammation by attracting more lymphocytes and provoking resident renal cells, leading to renal fibrosis and functional deterioration $(25,27-30)$.

TLRs, well-known for their roles in the recognition of pathogen-associated molecular patterns in innate immunity, are involved in the pathogenesis of IgAN (12). Serial studies have demonstrated that TLR9 is closely linked to Gd-IgA1 synthesis, cytokine secretion, tonsillectomy efficacy, and renal function in IgAN (31-35). TLR4 is elevated in patients with IgAN and correlated with disease severity (36, 37). TLR7 and TLR8 are actively involved in recognizing endogenous/exogenous single-stranded RNAs and initiating the inflammatory responses, including antibody synthesis and cytokine production (38). Despite high homology with respect to both sequence and function, TLR7 and TLR8 display different cell preferences and have diverse downstream biological effects $(39,40)$. TLR7 mostly targets B cells and plasmacytoid DCs for the synthesis of type I IFN, immunoglobulins, and cytokines, while TLR8 is more effective in inducing proinflammatory cytokines and chemokines in myeloid DCs and monocytes $(40,41)$. A previous study has reported that TLR7 mRNA levels are increased in peripheral blood mononuclear cells (PBMCs) of patients with IgAN (42). We also found that mRNA levels of TLR7 are strongly associated with mRNA levels of $\mathrm{B}$ cell activating factor belonging to TNF family $(B A F F)$ in PBMCs of patients with IgAN, indicating its potential impact on B cell functions in IgAN (43). Here, we present the first report to our knowledge demonstrating the involvement of TLR7 in IgAN with in-depth evidence. We explored the expression levels of TLR7 protein in kidney biopsy specimens from patients with IgAN and their correlations with renal function, histopathological parameters, and cytokine production, as evaluated by immunofluorescence staining and statistical analysis. Analysis of TLR7 in PBMCs and B cells, as well as EBV-immortalized B cells, revealed the increased TLR7 expression in B cells of patients with IgAN and the consequent impact on Gd-IgA1 synthesis via the TLR7-GALNT2 axis. Our study highlighted TLR7 as a potentially novel therapeutic target for treatment of IgAN.

\section{Results}

$T L R 7$ was highly expressed by intrarenal $C D 19^{+} B$ cells and correlated with renal functional injury in patients with $I g A N$. Renal biopsy specimens from non-CKD donors $(n=8)$ and patients with $\operatorname{IgAN}(n=89)$, minimal change disease $(\mathrm{MCD})(n=9)$, or membranous nephropathy $(\mathrm{MN})(n=11)$ were analyzed for TLR7 expression. Demographic and clinical characteristics were summarized in Table 1. As indicated in Supplemental Figure 1 (supplemental material available online with this article; https://doi.org/10.1172/jci.insight.136965DS1) and Figure 1A, TLR7 was strongly expressed in tubulointerstitial and periglomerular areas of renal biopsies from IgAN patients, as detected by 3 different TLR7 antibodies in immunofluorescence staining. However, TLR7 proteins were much less abundant in patients with MCD or MN (Figure 1A). The mean fluorescence intensities of TLR7 in kidney biopsies of patients with IgAN were about 5.0-fold higher than those of nonCKD donors $(P<0.01)$, 3.9-fold higher than those of patients with MCD $(P<0.01)$, and 2.0-fold higher than those of patients with $\mathrm{MN}(P<0.05)$ (Figure 1B). The mean intensities of renal TLR7 were higher in patients with impaired estimated glomerular filtration rate (eGFR) than in patients with normal eGFR $(P<$ 0.001) in IgAN (Figure 1C), indicating that TLR7 was more involved in patients with IgAN exhibiting renal damage. In contrast, expression of TLR8 was mostly observed in renal tubular cells (Figure 1D), and no significant difference was detected in non-CKD donors and IgAN patients (Figure 1E).

We found that TLR7 protein expression was enriched in renal B cells $\left(\mathrm{CD} 19^{+} / \mathrm{CD} 20^{+}\right.$) (Figure $2 \mathrm{~A}$ and Figure 3A). We detected a strong positive correlation between the mean intensities of TLR7 and CD19 proteins in kidneys of patients with $\operatorname{IgAN}(r=0.77, P<0.0001$, Figure 2B). Moreover, levels of the TLR7 and CD19 proteins exhibited negative correlations with eGFR levels $(r=-0.48$ and $P<0.0001$, and $r=-0.46$ and $P<0.0001$, respectively) (Figure 2, C and D) and positive associations with serum creatinine $(r=0.40$ and $P$ $<0.0001$, and $r=0.33$ and $P=0.0014$, respectively) (Figure 2, E and F). We also observed that TLR7 protein intensities were correlated with 24-hour proteinuria $(r=0.29, P=0.0049)$ in patients with IgAN (Figure 2G), 
Table 1. Clinical and demographic features of patients with IgAN and controls

\begin{tabular}{|c|c|c|c|c|c|c|}
\hline Feature & Non-CKD controls & IgAN & $\begin{array}{l}\text { Disease controls } \\
\text { (MN/MCD) }\end{array}$ & Healthy controls & IgAN & $\begin{array}{l}\text { Disease controls } \\
\text { (MN/MCD) }\end{array}$ \\
\hline Age (years) & $62.0 \pm 6.9$ & $32.5 \pm 9.6$ & $34.9 \pm 11.5$ & $33.2 \pm 9.8$ & $31.7 \pm 10.2$ & $35.2 \pm 13.3$ \\
\hline Serum creatinine $(\mu \mathrm{M})$ & $89.0 \pm 30.7$ & $104.3 \pm 57.9$ & $82.7 \pm 35.6$ & $69.3 \pm 37.3$ & $81.6 \pm 32.2$ & $75.4 \pm 32.7$ \\
\hline 24-h proteinuria (g) & n.d. & $1.18 \pm 1.26$ & $3.74 \pm 3.99^{\mathrm{B}, \mathrm{C}}$ & n.a. & $0.96 \pm 0.90$ & $4.08 \pm 3.56^{\mathrm{C}, \mathrm{D}}$ \\
\hline CKD II & n.a. & 13 & 1 & 0 & 6 & 1 \\
\hline CKD III & n.a. & 17 & 3 & 0 & 5 & 1 \\
\hline CKD IV & n.a. & 8 & 0 & 0 & 1 & 1 \\
\hline
\end{tabular}

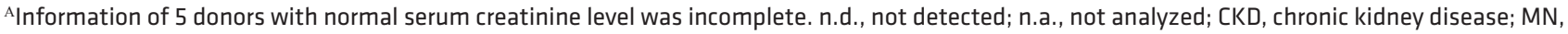
membranous nephropathy; $\mathrm{MCD}$, minimal change disease. ${ }^{\mathrm{B} P}<0.01$. ${ }^{\mathrm{C}}$ Disease controls versus patients with IgAN. Data were presented as median $\pm \mathrm{SD}$ and analyzed using 1-way ANOVA or Mann-Whitney $U$ test. ${ }^{D} P<0.001$.

but we did not detect a significant association between CD19 protein intensities and 24-hour proteinuria (data not shown). Additionally, the mean intensities of TLR7 and CD19 proteins were both significantly correlated with renal histopathological features, including tubular atrophy/interstitial fibrosis $(r=0.43$ and $P<0.001$, and $r=0.37$ and $P<0.001$, respectively), leukocyte infiltration ( $r=0.36$ and $P<0.001$, and $r=0.33$ and $P<0.01$, respectively), glomerular sclerosis ( $r=0.43$ and $P<0.001$, and $r=0.45$ and $P<0.001$, respectively), and C1q intensities ( $r=0.24$ and $P<0.05$, and $r=0.26$ and $P<0.01$, respectively), in patients with IgAN (Table 2).

The infiltration of $\mathrm{CD} 19^{+} \mathrm{B}$ cells in patients with IgAN varied from scarce infiltration (grade 0 ), or mild infiltration (grade 1), to dense infiltrates with $\mathrm{CD}^{+} \mathrm{T}$ cells (grade 2) in the kidney, as demonstrated in Supplemental Figure 2A. We also detected IgA-producing B cells in the tubulointerstitial area of patients with IgAN, though they were in small numbers (Supplemental Figure 2B and Figure 3A). These B cells in renal tissues of patients with IgAN produced high levels of IL-6 and IL-12 (Figure 3A). The presence of TLR7 in these cytokine-producing B cells was also confirmed (Supplemental Figure 2, C and D). Moreover, these cells were positive for the nuclear protein Ki67, suggesting in situ proliferation of B cells within the kidneys of patients with IgAN (Figure 3B).

TLR7 was upregulated in PBMCs and B cells from IgAN patients. Fresh PBMCs were isolated from 40 healthy donors, $46 \mathrm{IgAN}$ patients, and 26 disease controls (Supplemental Figure 3 ) and subjected to gene expression analysis by real-time PCR. Compared with healthy controls and disease controls, the relative mRNA levels of TLR7 and TLR 8 were significantly upregulated in patients with IgAN. However, mRNA levels of $O$-glycosyltransferases including GALNT2, C1GALT1, and COSMC in PBMCs were not altered in patients with IgAN (Figure 4A), although the mRNA levels of GALNT2 were significantly correlated with those of TLR7 in PBMCs of patients with $\operatorname{IgAN}(P<0.01)$ (Figure $4 \mathrm{~B})$.

We further isolated fresh $\mathrm{CD} 19^{+} \mathrm{B}$ cells from the peripheral blood and analyzed gene expression by real-time PCR. The mRNA levels of TLR7 and GALNT2 were significantly higher in patients with IgAN than in healthy controls (Figure 4C). TLR8, C1GALT1, and COSMC mRNA were detected in B cells; however, no significant differences in their gene expression levels were observed between patients with IgAN and healthy controls (Figure 4C).

We next immortalized B cells from patients with IgAN with EBV (as shown in Supplemental Figure 4) and examined TLR7, IgA, and GALNT2 expression. The protein levels of TLR7 and IgA were significantly increased in EBV-immortalized B cells from patients with IgAN compared with in those from healthy controls (Figure 4, D and E). For the protein levels of GALNT2, they tend to be increased in EBV-immortalized B cells from patients with IgAN, though there is no statistical significance (Figure 4, D and E). The protein levels of TLR7 and GALNT2 were significantly associated in EBV-immortalized B cells from patients with $\operatorname{IgAN}(P<0.01)$ (Figure 4F).

In vitro activation of TLR7 boosted production of IgA1 and Gd-IgA1 in PBMCs from patients with IgAN. R848, an agonist of both TLR7 and TLR8, was used to stimulate PBMCs in vitro to promote the proliferation of 
A

Non-CKD-1

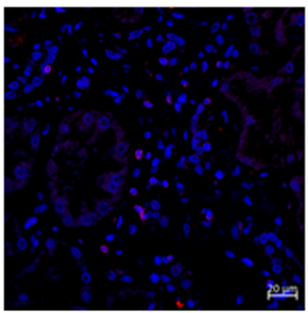

$\lg \mathrm{AN}-1$

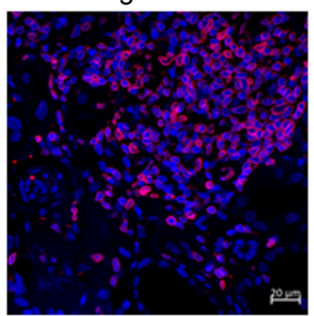

MCD-1

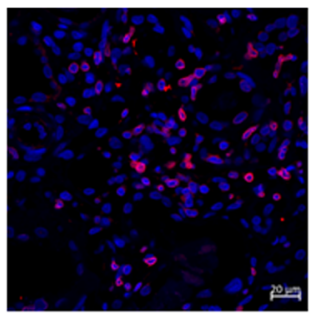

B

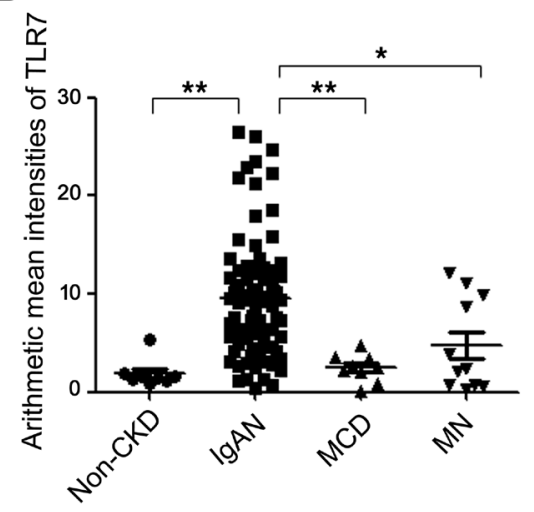

C

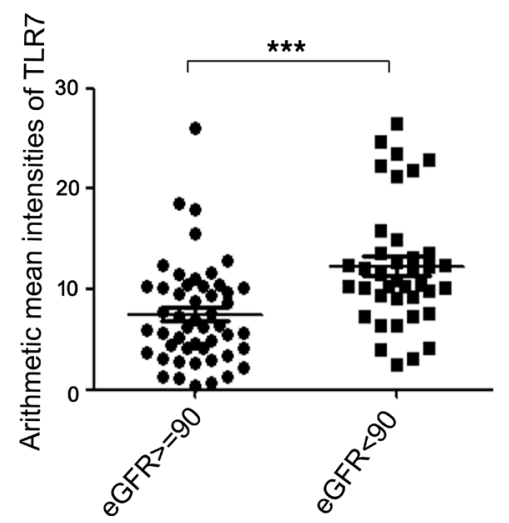

D

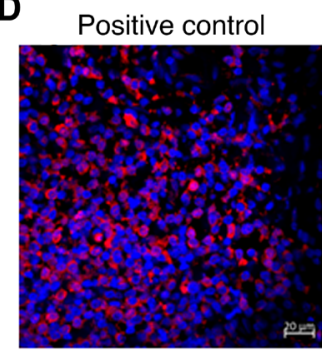

E

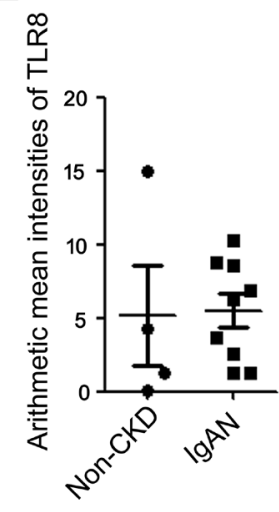

pen
Non-CKD-4

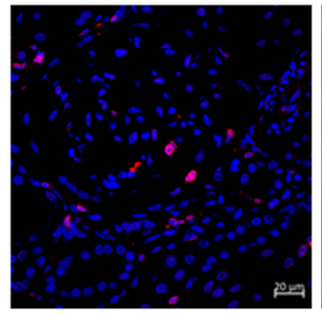

$\lg \mathrm{AN}-3$

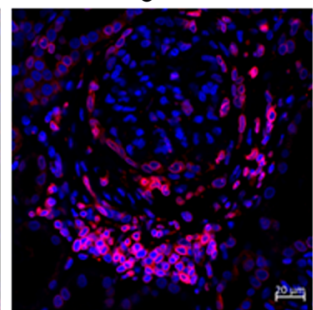

$\mathrm{MN}-1$

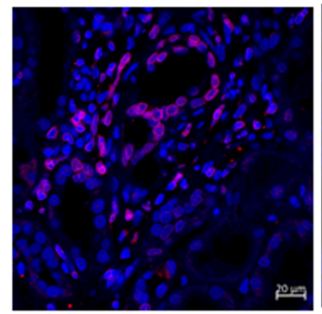

ine

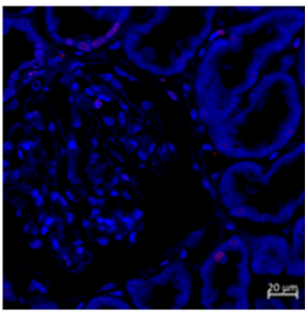

$\lg \mathrm{AN}-4$

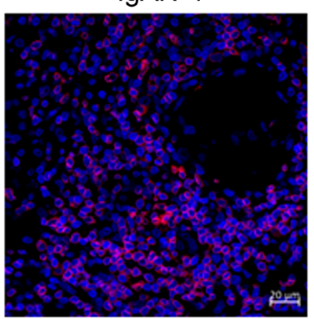

MN-2

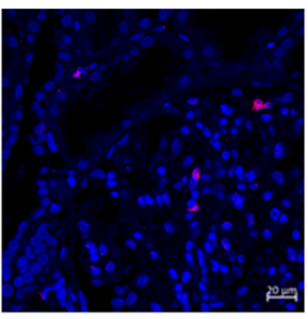

Non-CKD-1

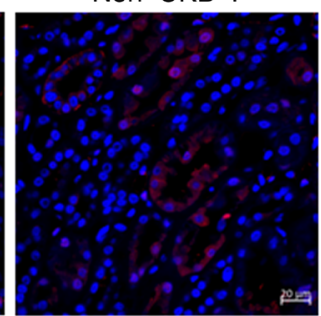

$\lg \mathrm{AN}-1$

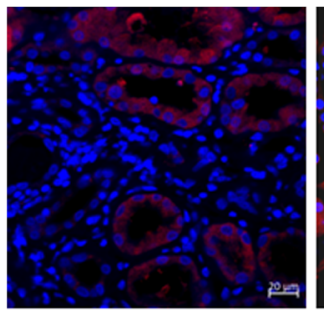

Non-CKD-5

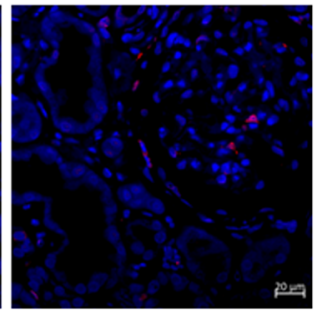

IgAN-5

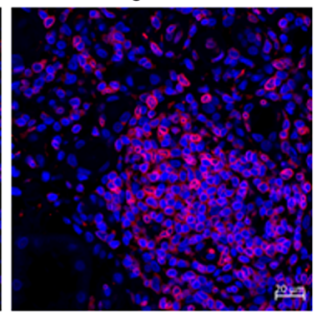

MN-3

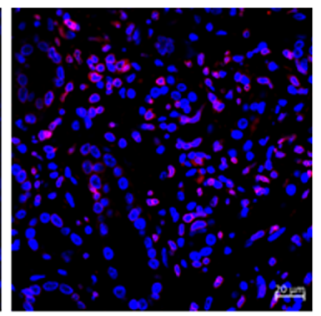

Non-CKD-2

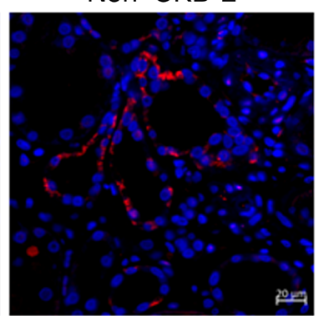

$\lg A N-2$

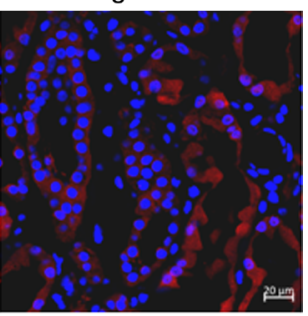


Figure 1. Enriched TLR7 proteins presented in kidneys of patients with IgAN. (A) Representative immunofluorescence images of TLR7 proteins (red) in paraffin-embedded renal biopsy specimens from patients with IgAN and controls. Nuclei were counterstained with DAPI (blue). (B) Quantification of mean fluorescence intensities of TLR7 proteins in kidneys of patients with IgAN $(n=89)$, compared with non-CKD donors $(n=8)$, patients with MCD $(n=9)$, and patients with MN $(n=11)$. Data were presented as median \pm SEM and analyzed using 1-way ANOVA with Bonferroni's correction. (C) Comparison of mean fluorescence intensities of intrarenal TLR7 proteins in patients with IgAN with different grades of eGFR. (D) Representative immunofluorescence images of TLR8 proteins (red) in IgAN and non-CKD patients, using human tonsil specimen as positive control. (E) Quantification of mean fluorescence intensities of TLR8 proteins in kidneys of patients with IgAN $(n=9)$ compared with non-CKD donors $(n=4)$. Non-CKD, donors without chronic kidney diseases; IgAN, IgA nephropathy; MCD, minimal change disease; MN, membranous nephropathy. Data were analyzed using 2-tailed unpaired Student's $t$ test for $\mathbf{C}$ and $\mathbf{E}$. Significance levels are set at ${ }^{*} P<0.05,{ }^{*} P<0.01,{ }^{* *} P<0.001$. Scale bars: $20 \mu \mathrm{m}$.

$\mathrm{CD}^{+} 9^{+} \mathrm{B}$ cells (Supplemental Figure 5A) and the secretion of IgA1 antibodies (Supplemental Figure 5B). After 12 days of culture, ELISA showed that TLR7/8 activation led to substantially enhanced IgA1 secretion from PBMCs of patients with IgAN $(1318 \pm 257 \mathrm{ng} / \mathrm{mL})$, as compared with healthy controls (481 \pm 63 $\mathrm{ng} / \mathrm{mL}, P<0.001)$ and disease controls $(591 \pm 135 \mathrm{ng} / \mathrm{mL}, P<0.05)$ (Figure 5A, left panel). In addition, Western blot analysis also detected that in vitro activation of TLR7/8 significantly promoted PBMCs to produce more intracellular IgA antibodies from patients with $\operatorname{IgAN}$, as compared with healthy donors and disease donors (Supplemental Figure 6, A and B). With respect to Gal deficiency, IgA1 antibodies secreted from R848-activated PBMCs displayed significantly higher helix aspersa (HAA) lectin binding $\left(\mathrm{OD}_{450} \mathrm{HAA} /\right.$ $\mathrm{OD}_{450} \mathrm{IgA1}$ ) in the group of patients with IgAN than in healthy controls (Figure 5A, right panel). Furthermore, the concentrations of secreted IgA1 molecules in PBMCs cultures after TLR7/8 activation were significantly correlated with serum IgA concentration $(P<0.05)$ in patients with IgAN (Figure 5B).

To further dissect the effects of TLR7 and TLR8 on production of Gd-IgA1, we employed inhibitors for TLR7 and TLR8 in PBMC culture experiments. As indicated in Figure 5C, the synthesis of IgA1 was diminished in 11 of 13 donors, while Gal deficiency of IgA1 was alleviated in 7 of 10 donors when using a TLR7 inhibitor (Zinc 4756232) in R848-treated PBMCs. Moreover, when using the TLR7-selective agonist loxoribine to assess the effect of the TLR7 inhibitor, IgA1 secretion was reduced in 12 of 13 donors $(P<$ $0.05)$, and Gal deficiency of IgA1 was alleviated in 7 of 8 donors $(P<0.05)$ (Figure 5D). In contrast, blockade of TLR8 with a specific inhibitor, CU-CTP8m (CAS 125079-83-6), did not show consistent inhibitory effects on IgA1 and Gd-IgA1 synthesis upon R848 activation (Figure 5E).

Increased expression of the O-glycosyltransferase GALNT2 in activated B cells of patients with IgAN. To investigate the impact of TLR7/8 activation on gene expression of $O$-glycosyltranferases, PBMCs after TLR7/8 activation were submitted to real-time PCR and Western blot analysis. On day 4, mRNA levels of GALNT2 were significantly increased in activated PBMCs from patients with IgAN compared with healthy controls, which resulted in a significant decrease in the ratio of C1GALT1/GALNT2 (Supplemental Figure 6C). At the protein level, the C1GALT1/GALNT2 ratio was also significantly lower in patients with IgAN than in controls after 12 days of culture with R848 (Supplemental Figure 6, A and B).

The mRNA levels of TLRs and O-glycosyltransferases were explored in B cells and non-B cells from healthy donors after R848 stimulation. As indicated in Figure 6A, mRNA levels of TLR7 were initially much higher in B cells than in non-B cells (0 hours) and were dramatically upregulated in CD19+ B cells but not CD19 cells after 6 days of culture. The expression pattern of GALNT2 was highly similar to that of TLR7 in B cells, with a significant increase in $\mathrm{CD} 19^{+} \mathrm{B}$ cells and low levels in non-B cells after R848 stimulation for 6 days. $T L R 8$ was enriched in non-B cells rather than B cells at 0 hours and retained low expression levels in B cells after R848 activation. The mRNA levels of C1GALT1 were higher in B cells than in non-B cells at 0 hours, but C1GALT1 expression was substantially suppressed in B cells after R848 stimulation.

Since B cells are responsible for IgA1 synthesis and its $O$-glycan modification, R848-activated CD19 ${ }^{+}$ cells were sorted for analysis of C1GALT1 and GALNT2 expression after 9 days of culture. CD19+ B cells demonstrated significantly higher protein levels of GALNT2 in patients with IgAN than those in healthy controls and disease controls, and a similar pattern was not observed in non-B (CD19-) cells (Figure 6B). The protein levels of C1GALT1 in B cells did not differ significantly among groups. Furthermore, the relative C1GALT1/GALNT2 ratio was significantly lower in B cells of patients with IgAN than in healthy controls and disease controls after R848 stimulation (Figure 6B).

TLR7-GALNT2 axis modulated IgA1 O-glycosylation in patients with IgAN. Adenoviruses respectively expressing TLR7 or GFP were transfected into HEK293T cells to study the regulatory effect on gene expression of GALNT2. As shown in Figure 7, overexpression of TLR7 in HEK293T cells led to increased protein levels of GALNT2 in the presence or absence of R848, without significant change in expression 
A

TLR7

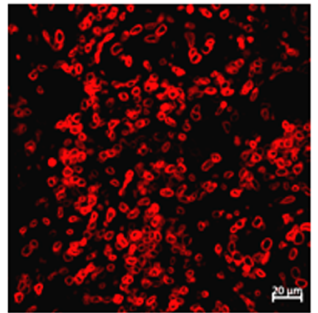

TLR7

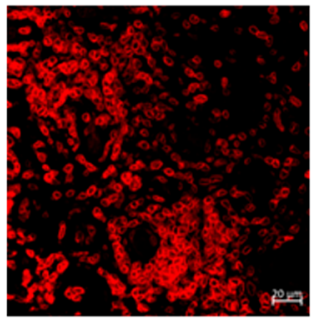

B

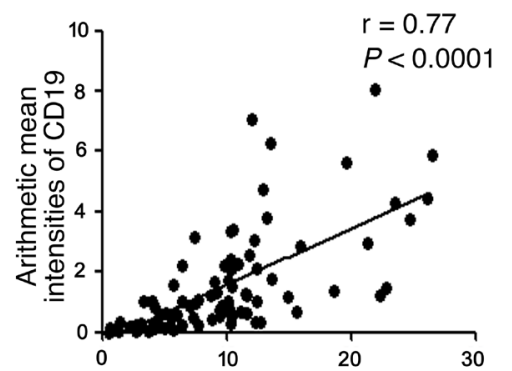

Arithmetic mean intensities of TLR7

$\mathbf{E}$

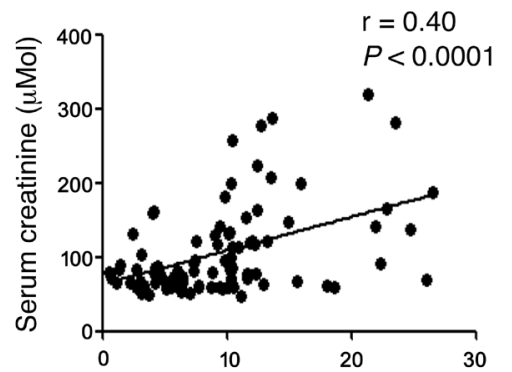

Arithmetic mean intensities of TLR7
CD20

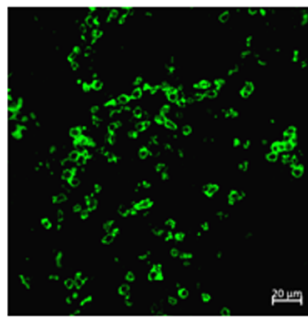

CD19

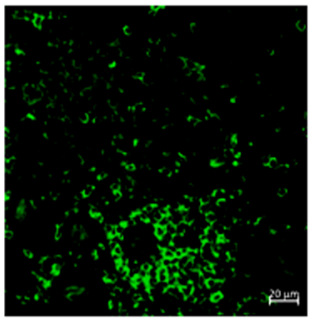

C

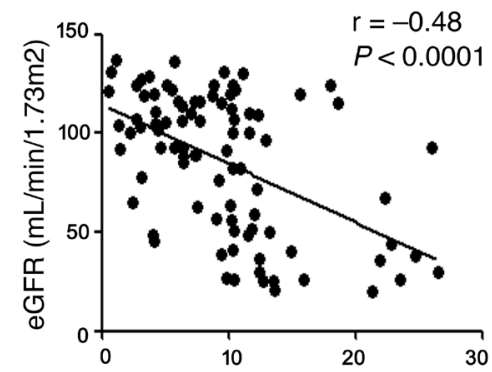

Arithmetic mean intensities of TLR7

$\mathbf{F}$

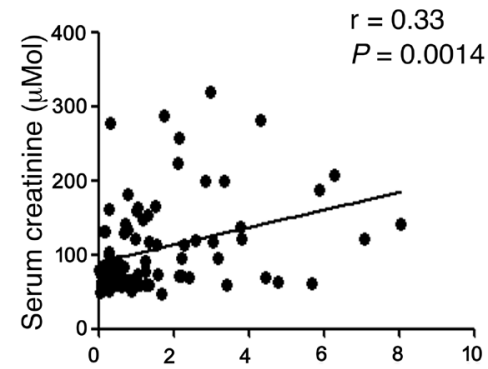

Arithmetic mean intensities of CD19

DAPI
Merge
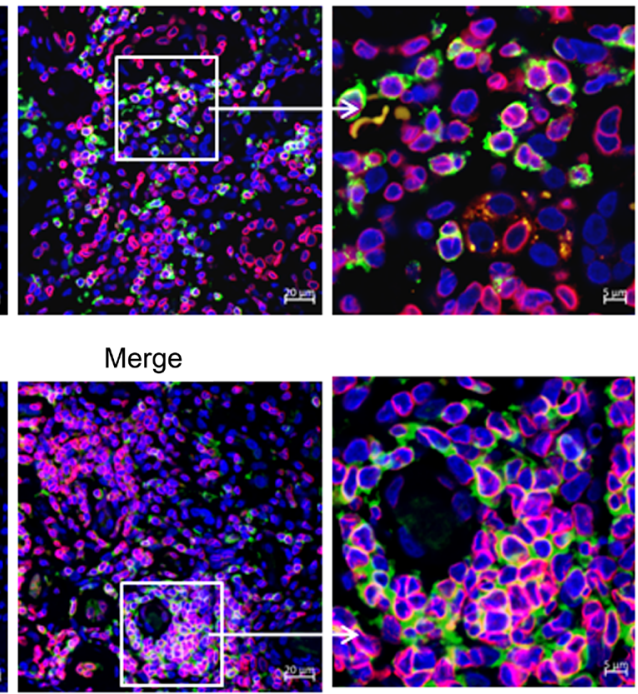

D

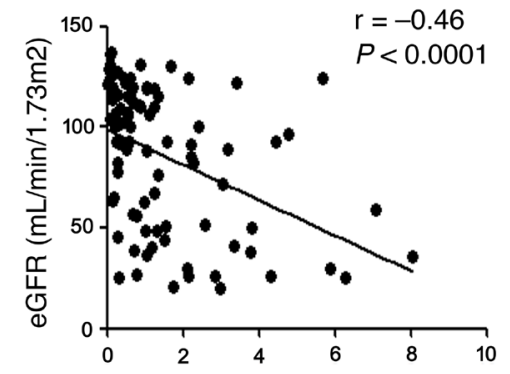

Arithmetic mean intensities of CD19

G

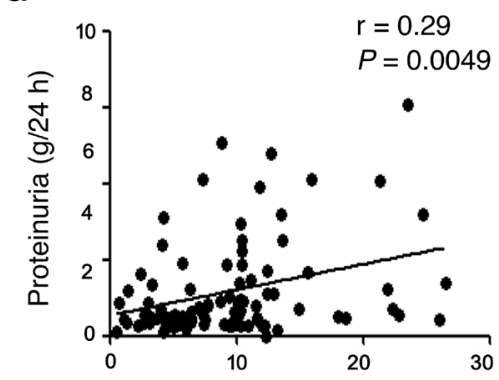

Arithmetic mean intensities of TLR7

Figure 2. Enhanced abundance of TLR7 proteins and B cells was linked to renal function and histopathological features in patients with IgAN. (A) Representative immunofluorescence images of TLR7 proteins in $\mathrm{CD}_{19}{ }^{+}$and $\mathrm{CD}_{20} \mathrm{O}^{+}$cells in paraffin-embedded renal specimens from patients with IgAN $(n=7)$. Scale bars: $20 \mu \mathrm{m}$. (B) Intensity values of TLR7 and CD19 proteins in renal tissues exhibited significant correlation in patients with IgAN. (C and D) Estimated glomerular filtration rates (eGFRs) were significantly correlated with fluorescence intensities of TLR7 (C) and CD19 proteins (D) in patients with IgAN. (E and F) Serum creatinine levels were significantly correlated with fluorescence intensities of TLR7 (E) and CD19 proteins (F) in patients with IgAN. (C) Fluorescence intensities of TLR7 proteins were significantly associated with 24-hour proteinuria in patients with IgAN. $n=89$. Data were analyzed using Spearman's correlations.

of C1GALT1. Both DAKIKI B cells and EBV-immortalized B cells expressed endogenous TLR7. When using siRNA to knock down TLR7 expression, GALNT2 protein levels were reduced in both IgAN EBV-immortalized B cells (Figure 8A) and DAKIKI B cells (Figure 8B), but the expression of C1GALT1 was not affected.

To further evaluate the effect of GALNT2 on $O$-glycan modification of IgA1 molecules, we delivered adenoviruses expressing GALNT2 into EBV-immortalized B cell lines. As indicated in Figure 8C, the upregulation of GALNT2 was confirmed in IgAN EBV-immortalized B cells after adenovirus transfection. 
A

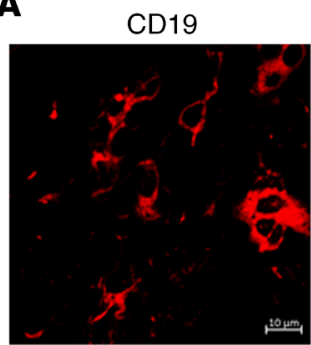

CD19

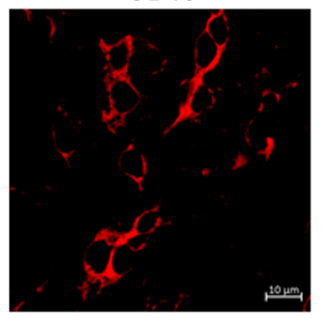

CD19

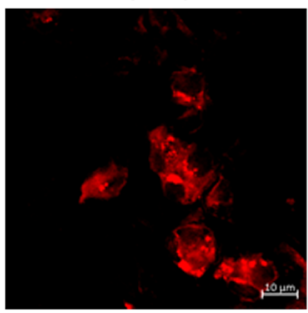

CD19

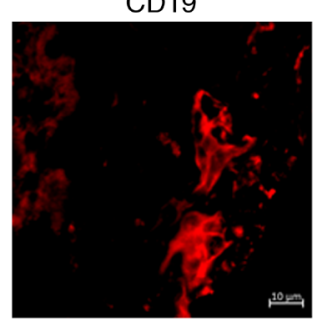

B
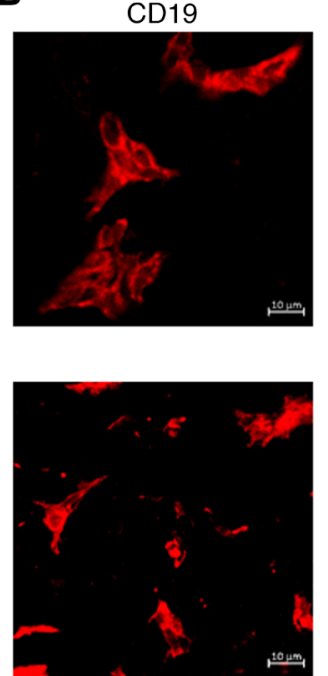

TLR7

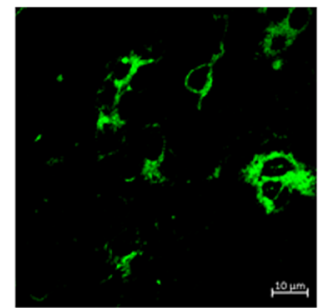

$\lg A$

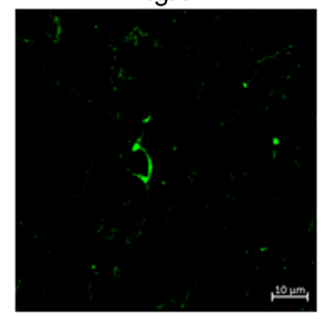

IL-6

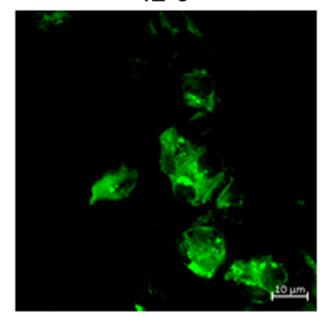

IL-12

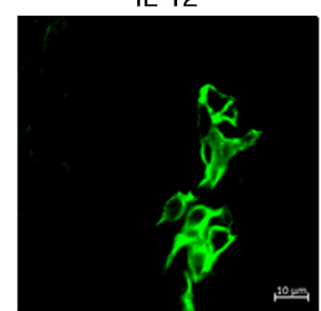

Ki67
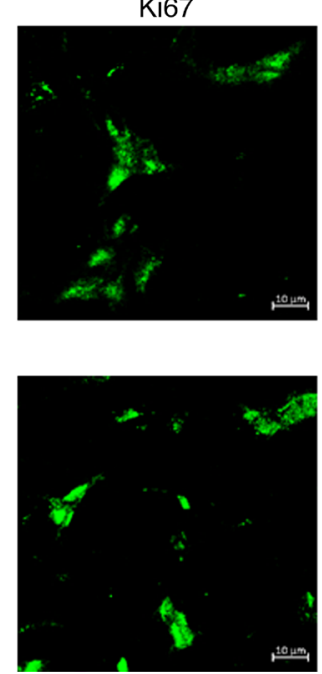

DAPI

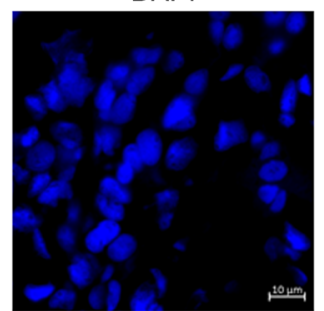

DAPI

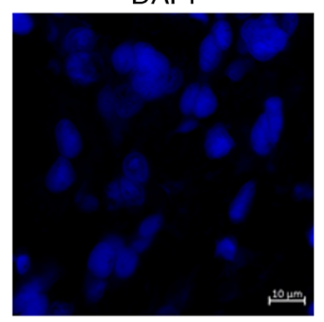

DAPI

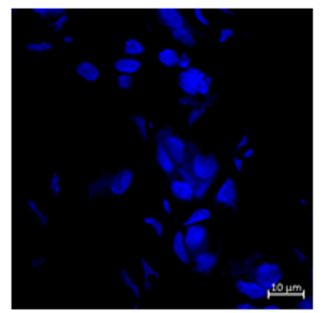

DAPI

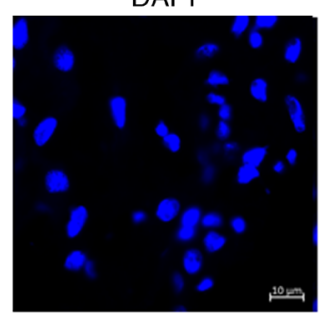

DAPI
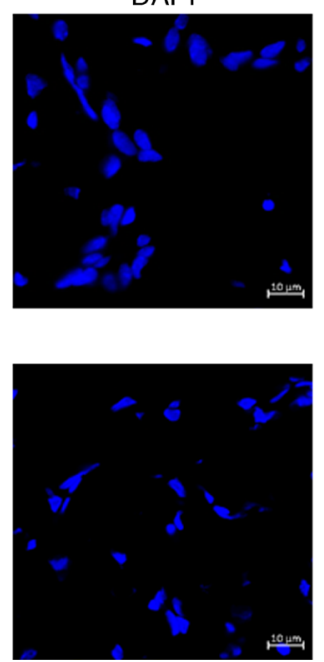

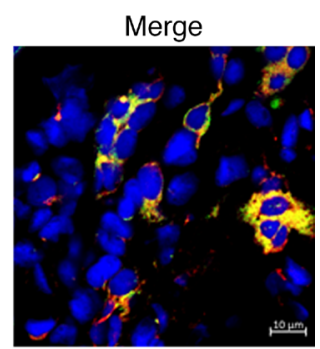

Merge

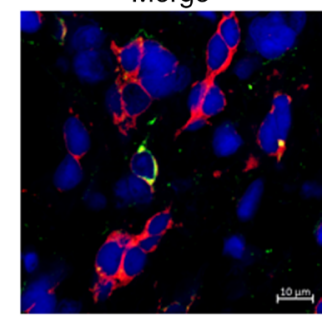

Merge

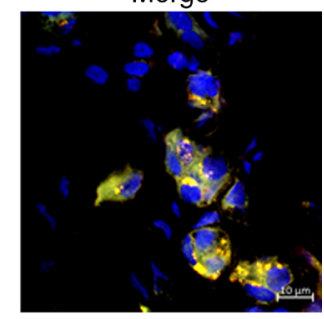

Merge

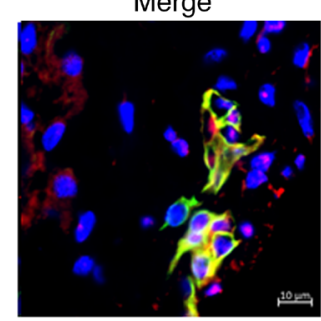

Merge
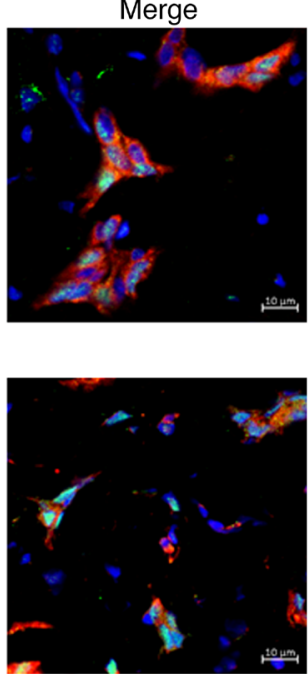

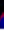

Figure 3. The production of IL-6 and IL-12 from $B$ cells in renal tissue of patients with IgAN. (A and B) Representative confocal images for fluorescence staining of TLR7, IgA, IL-6, IL-12 (A), and Ki67 (B) proteins in intrarenal CD19+ $B$ cells of patients with IgAN from frozen sections of renal biopsy specimens $(n=4)$. Scale bars: $10 \mu \mathrm{m}$. 
Table 2. Association of fluorescence intensities of intrarenal TLR7 and CD19 proteins with histopathological parameters in patients with IgAN

\begin{tabular}{|c|c|c|c|c|c|}
\hline \multirow[t]{2}{*}{ Feature } & \multicolumn{2}{|c|}{ Renal TLR7 protein fluorescence intensities } & \multirow[t]{2}{*}{ Feature } & \multicolumn{2}{|c|}{ Renal CD19 protein fluorescence intensities } \\
\hline & Spearman $r$ & $P$ value & & Spearman $r$ & $P$ value \\
\hline $\begin{array}{l}\text { Mesangial } \\
\text { hypercellularity }\end{array}$ & -0.03 & 0.73 & $\begin{array}{l}\text { Mesangial } \\
\text { hypercellularity }\end{array}$ & -0.02 & 0.83 \\
\hline $\begin{array}{l}\text { Tubular atrophy/ } \\
\text { Interstitial fibrosis }\end{array}$ & 0.43 & $0.0002^{\mathrm{A}}$ & $\begin{array}{l}\text { Tubular atrophy/ } \\
\text { Interstitial fibrosis }\end{array}$ & 0.37 & $0.0003^{\mathrm{A}}$ \\
\hline $\begin{array}{l}\text { Global glomerular } \\
\text { sclerosis (\%) }\end{array}$ & 0.43 & $<0.0001^{A}$ & $\begin{array}{l}\text { Global glomerular } \\
\text { sclerosis (\%) }\end{array}$ & 0.45 & $<0.0001^{A}$ \\
\hline Renal IgA intensity & 0.11 & 0.28 & Renal IgA intensity & -0.07 & 0.48 \\
\hline Renal IgG intensity & 0.013 & 0.90 & Renal IgC intensity & -0.004 & 0.96 \\
\hline Renal IgM intensity & 0.03 & 0.77 & Renal IgM intensity & 0.001 & 0.99 \\
\hline Renal C1q intensity & 0.24 & $0.024^{\mathrm{C}}$ & Renal C1q intensity & 0.26 & $0.0013^{B}$ \\
\hline
\end{tabular}

The increased expression of GALNT2 did not significantly influence the protein levels of C1GALT1. In the supernatant, the secretion of IgA1 was not significantly changed by the overexpression of GALNT2 (Figure 8D, upper panel). However, the production of Gd-IgA1 was significantly higher in GALNT2-expressing B cells than in GFP-expressing controls $(19.6 \pm 2.6$ versus $2.6 \pm 1.5 \mathrm{ng} / \mathrm{mL}, P<0.001$, Figure $8 \mathrm{D}$, lower panel), which mimicked the Gal deficiency of IgA1 molecules in patients with IgAN.

\section{Discussion}

B cell infiltration in the tubulointerstitial space of the kidney has been observed in patients with IgAN $(24,44)$. Pei et al. detected tertiary lymphoid organ neogenesis in $37.5 \%$ of kidney biopsies from patients with IgAN and found that intrarenal plasma cells have the ability to produce IgG antibodies (44). We confirmed that the renal infiltration of $\mathrm{B}$ cells and $\mathrm{T}$ cells varied from scarce infiltration to nodule-like structure in patients with IgAN. Our study found that TLR7 expression was enriched in infiltrated $\mathrm{CD} 19^{+} \mathrm{B}$ cells in kidneys of patients with $\operatorname{IgAN}(n=89)$ and was closely related to kidney function and renal histopathology, indicating that TLR7 expression in B cells is involved in the pathogenesis and progression of IgAN. Ciferska et al. recently detected positive staining of TLR7 in kidney of patients with IgAN but did not find direct correlation between TLR7 intensities and clinical parameters, which may be explained by the small sample size $(n=34)$ and the use of less sensitive technology (i.e., IHC rather than immunofluorescence) (45).

In human B cells, it is well established that the activation of TLR7 can activate the MyD88-dependent signaling pathway, which results in B cell expansion, immunoglobulin secretion, and synthesis of cytokines, including IL-6, IL-12, and IL-1 $\beta$ (46-49). We observed high expression of IL-6 and IL-12 in renal $\mathrm{TLR} 7^{+} \mathrm{B}$ cells of patients with IgAN, and these B cells were even capable of proliferation. It has been confirmed that IL-6 is elevated in renal tissues and is a prognostic factor in patients with IgAN $(50,51)$. Meanwhile, serum and renal levels of IL-12 are elevated in patients with IgAN $(52,53)$. The downstream effects of IL-6 include promoting the proliferation of mesangial cells and the apoptosis of podocytes in vitro, as well as regulating endothelial dysfunction and extracellular matrix production, ultimately resulting in renal fibrosis and tissue destruction (54-58). IL-12 drives renal injury by inducing the accumulation of lymphocytes, the secretion of IFN- $\gamma$, and the formation of crescents in mice (59, 60). Consistently, we found that the intensities of both intrarenal TLR7 and CD19 proteins were significantly correlated with leukocyte infiltration and renal fibrosis in IgAN patients. Collectively, these data suggest that TLR7-expressing B cells are critical players in renal inflammation of IgAN, mediating by proinflammatory cytokines and downstream effects. 
A

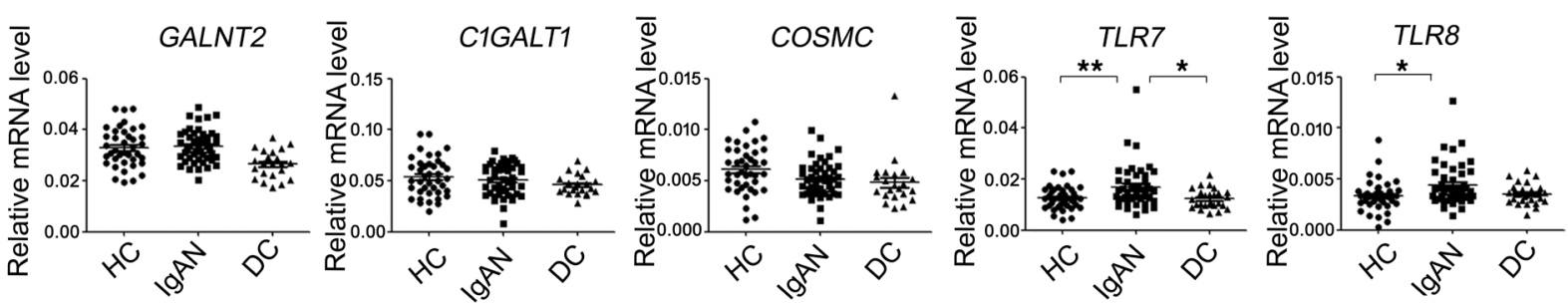

B

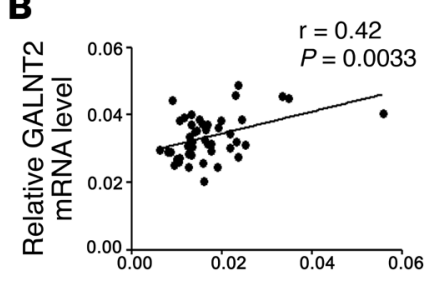

Relative TLR7 mRNA level
C

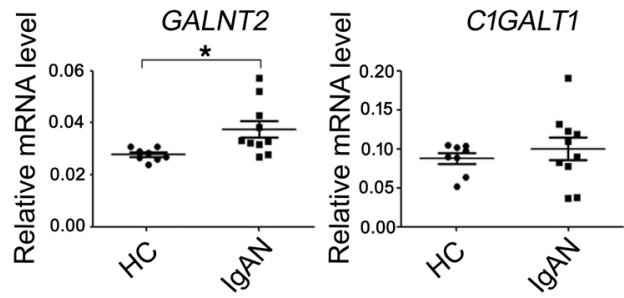

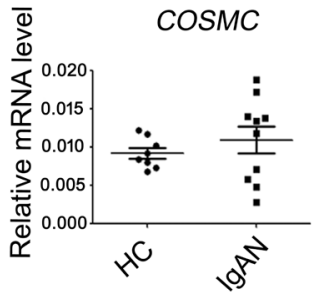

TLR8
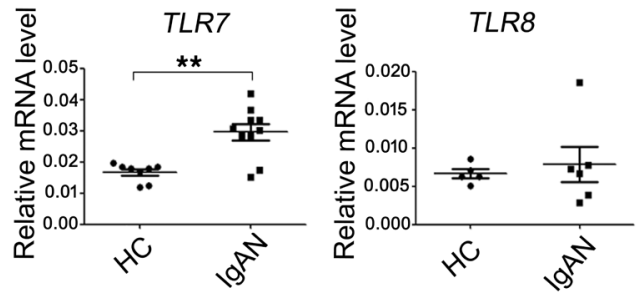

D

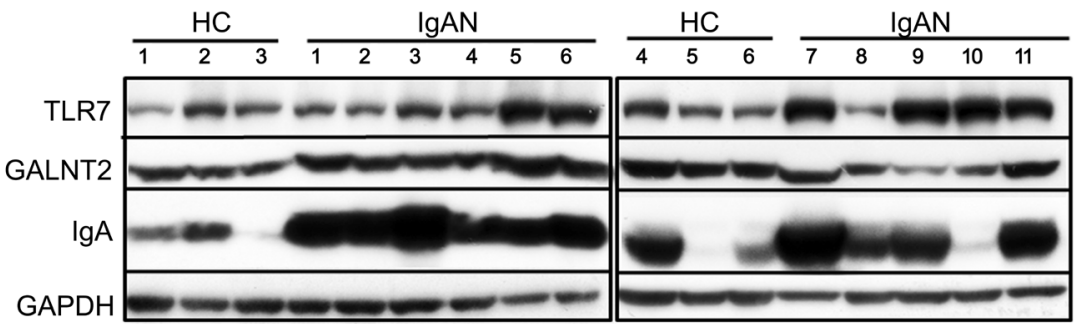

$\mathbf{E}$

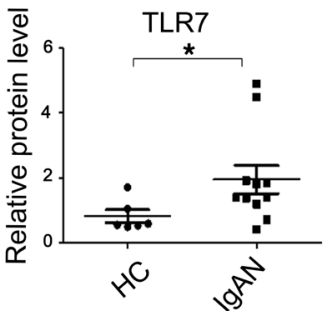

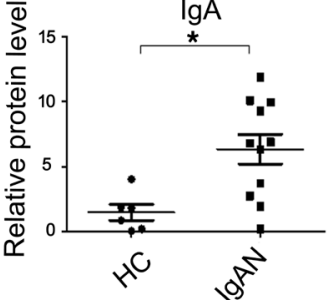

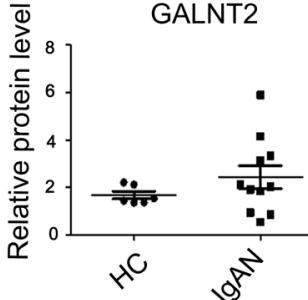

$\mathbf{F}$

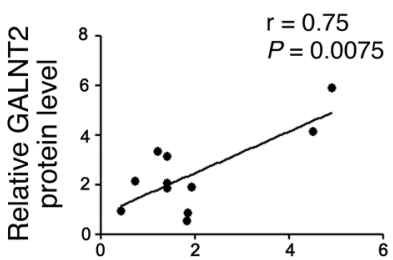

Relative TLR7 protein level

Figure 4. Increased expression of TLR7 in peripheral B cells of patients with IgAN. (A) The relative mRNA levels of TLRs and 0 -glycosyltransferases in PBMCs. PBMCs from patients with IgAN $(n=46)$, healthy controls $(n=40)$, and disease donors $(n=26)$ were prepared by Ficoll-Paque density centrifugation and subjected to quantitative PCR analysis for target genes, using GAPDH as internal controls. Statistical significance was determined by 1-way ANOVA with Bonferroni's correction. (B) The association analysis of relative mRNA levels of TLR7 and CALNT2 in PBMCs of IgAN patients. (C) The relative mRNA levels of TLRs and O-glycosyltransferases in sorted fresh CD19+ B cells from patients with IgAN $(n=6-10)$ and healthy controls $(n=5-8)$. (D) The expression of TLR7, GALNT2, and IgA proteins in EBV-immortalized B cells from patients with IgAN $(n=11)$ and healthy donors $(n=6)$, as detected by Western blot. (E) Quantification and statistical data for $\mathbf{D}$, using GAPDH as internal control. (F) The association analysis of relative protein levels of TLR7 and GALNT2 in EBV-immortalized B cells derived from IgAN patients. HC, healthy controls; IgAN, patients with IgAN; DC, disease controls (MCD/MN). Data were presented as median \pm SEM and analyzed by 2-tailed unpaired Student's $t$ test ( $\mathbf{C}$ and $\mathbf{E}$ ) and Pearson correlation analysis (B and $\mathbf{F}) .{ }^{*} P<0.05,{ }^{*} P<0.01$.

Studies involving animal models have shown that TLR7 is involved in the pathogenesis of glomerulonephritis, including lupus nephritis and diabetic nephropathy; agonists of TLR7 aggravate glomerulonephritis symptoms in both diseases, while the blockade of TLR7 could alleviate renal injury (61-64). High renal TLR7 expression has been detected in both lupus-prone mice and a diabetic animal model, and it is 
A

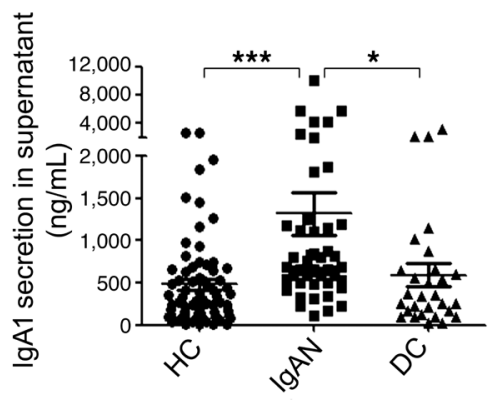

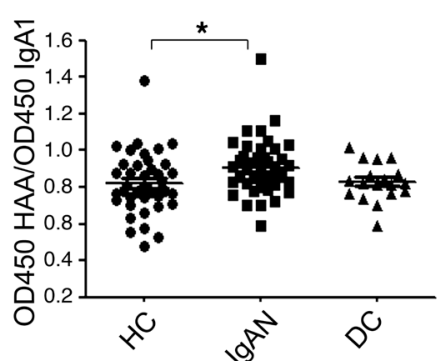

B

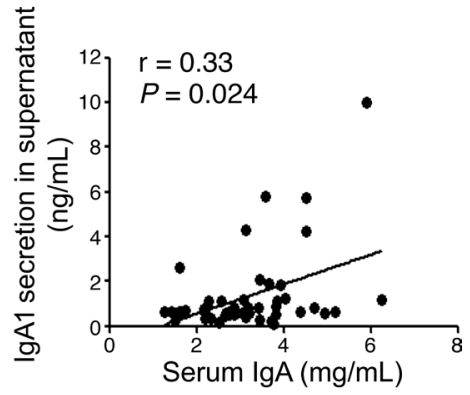

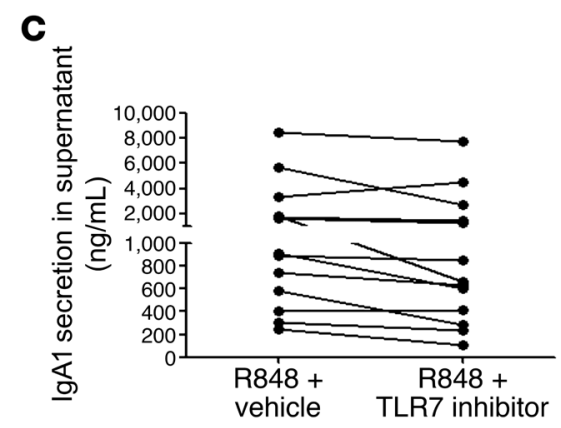

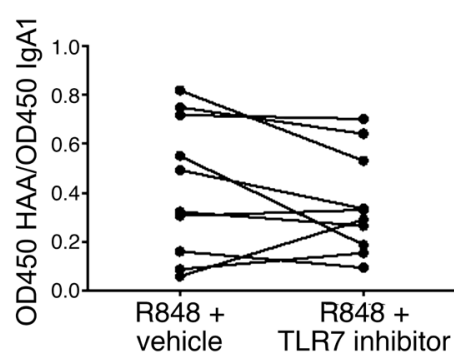

D
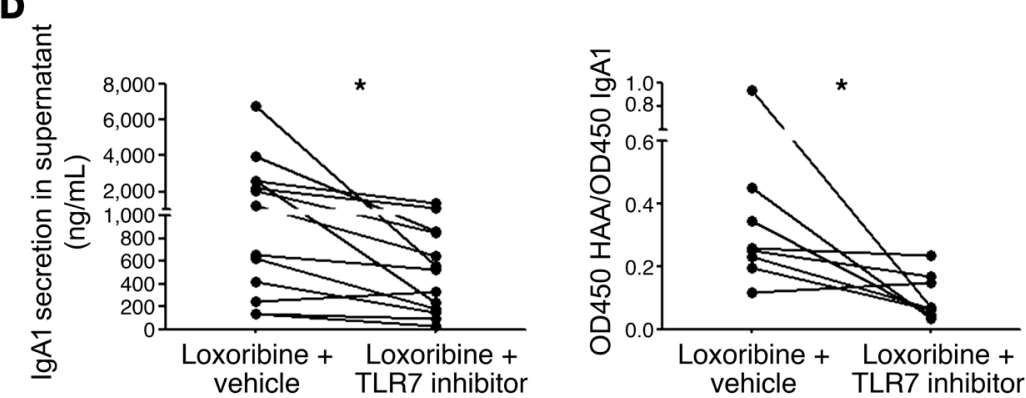

$\mathbf{E}$
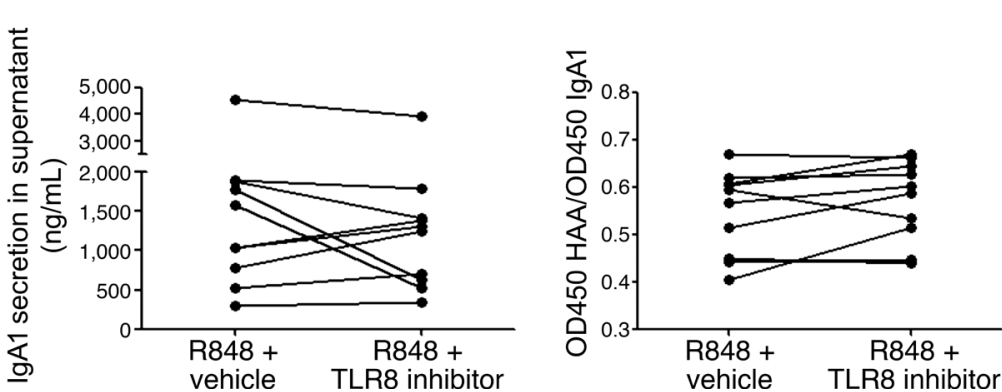

Figure 5. Secretion of IgA1 and Gd-IgA1 was enhanced in PBMCs of patients with IgAN via activation of TLR7/8 in vitro. (A) PBMCs from healthy controls (HC, $n=72$ ), patients with IgAN (IgAN, $n=49$ ), and disease controls (DC, $n=29)$ were purified and cultured with R848 $(5 \mu \mathrm{g} / \mathrm{mL})$ for 12 days. Culture supernatant was collected and measured for IgA1 content and IgA1 galactose deficiency by sandwich ELISA and HAA lectin binding assay, respectively. One-way ANOVA with Bonferroni's post hoc test. ${ }^{*} P<0.05$, ${ }^{* *} P<0.001$. (B) The correlation of IgA1 secretion in culture supernatant after R848 stimulation and serum IgA from patients with IgAN was analyzed with Spearman $r$ test. (C) PBMCs culture $(n=13)$ activated by R848 $(5 \mu \mathrm{g} / \mathrm{mL})$ in the presence or absence of TLR7 inhibitor $(100 \mu \mathrm{M})$. (D) PBMCs culture $(n=13)$ activated by loxoribine $(500 \mu \mathrm{M})$ in the presence or absence of TLR7 inhibitor $(100 \mu \mathrm{M})$. (E) PBMCs culture $(n=10)$ activated by R848 treatment $(5 \mu \mathrm{g} / \mathrm{mL})$ with or without TLR8 inhibitor CU-CPT8m $(1 \mu \mathrm{M})$. Samples with IgA1 secretion lower than $250 \mathrm{ng}$ / $\mathrm{mL}$ were not included in HAA lectin binding assay. ${ }^{*} P<0.05$ by 2 -tailed paired Student's $t$ test for $\mathbf{C}$-E.

closely related to the production of proinflammatory cytokines (e.g., IL-6, IL-1 $\beta$, TNF- $\alpha$, and IL-12) and consequent renal injury $(61,63-65)$. The infiltration of B cells and increased expression of TLR7 has been demonstrated in kidney specimens of patients with lupus nephritis $(25,26,66)$. Based on this evidence, we conjecture that TLR7 contributes to B cell-mediated renal inflammation in glomerulonephritis such as lupus nephritis and IgAN. However, limited data are available on the roles of TLR7 in human glomerulonephritis, and further studies are urgently needed to address this shortfall. 
A
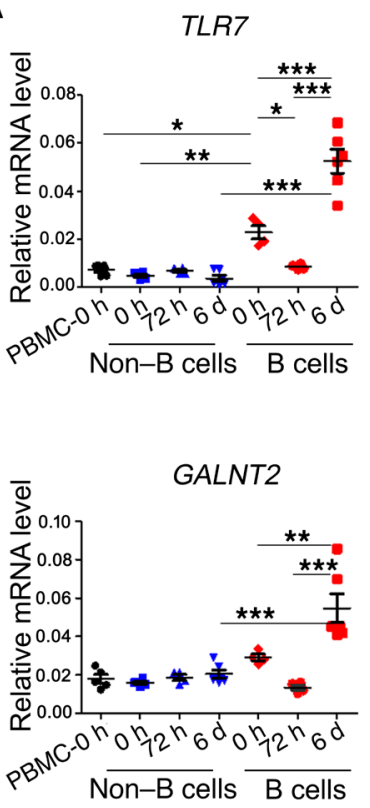
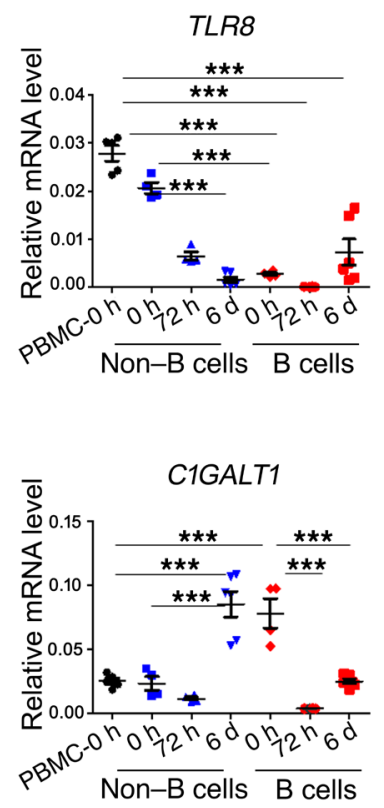

B
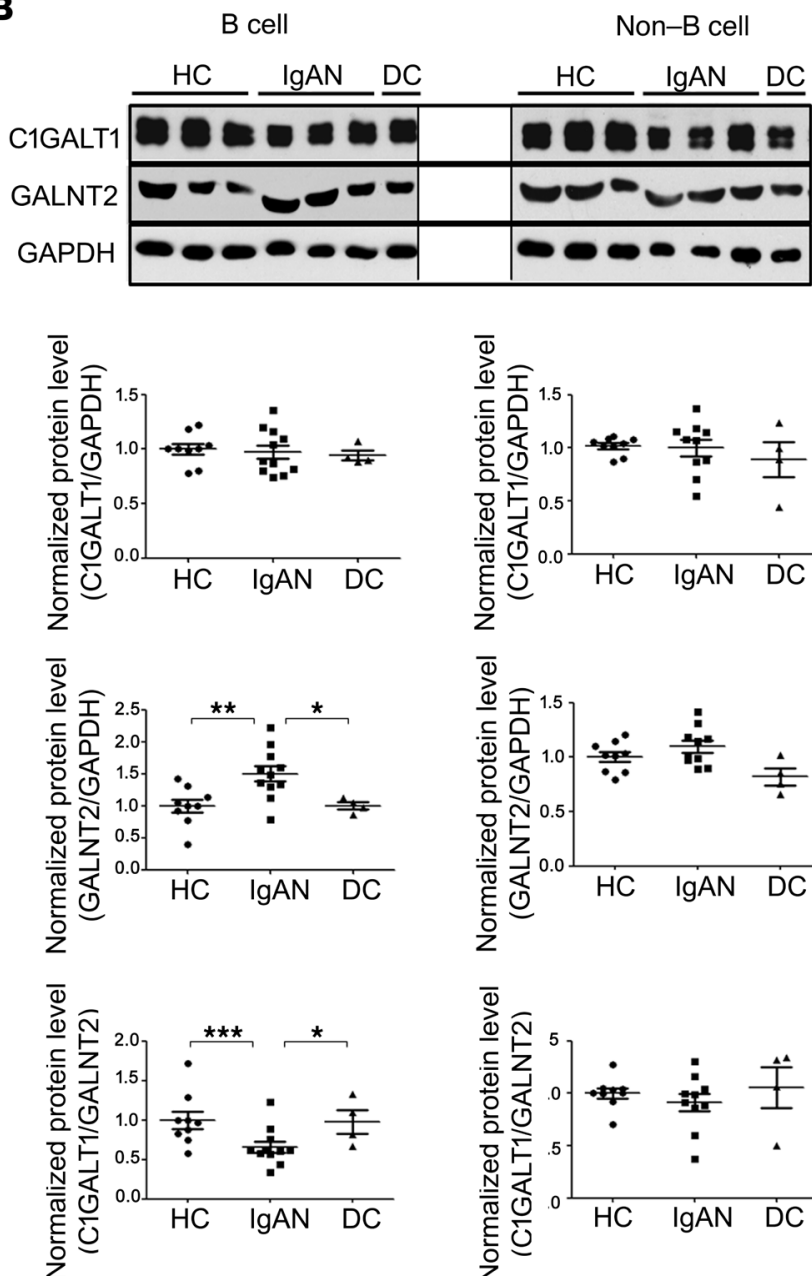
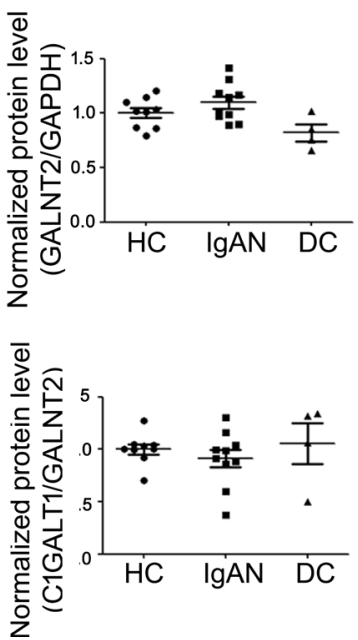

Figure 6. Protein levels of GALNT2 were upregulated in B cells of patients with IgAN after TLR7/8 activation in vitro. (A) PBMCs from healthy donors $(n=4-6)$ were purified and cultured with R848 $(5 \mu \mathrm{g} / \mathrm{mL})$ for 6 days. Cell samples were collected at different time points and sorted into B cells (CD19+) cells and non-B cells (CD19-) by flow cytometry. Cells are subjected to mRNA extraction, CDNA synthesis, and quantitative PCR analysis for target genes using GAPDH as housekeeping gene. (B) Representative Western blot images of protein expression in B cells and non-B cells from R848-activated PBMCs and quantification. PBMCs from donors were prepared and cultured with R848 (5 $\mu \mathrm{g} / \mathrm{mL})$. Cells were collected after 9 days and sorted into B cells (CD19+) and non-B cells (CD19-). Cells are subjected to proteins extraction and Western blot analysis. HC, healthy controls ( $n=35$, combined as 9 samples); IgAN, patients with IgAN ( $n=31$, combined as 11 samples); DC, disease controls (MCD/MN) ( $n=15$, combined as 4 samples). Data were analyzed by 1-way ANOVA with post hoc test. ${ }^{*} P<0.05,{ }^{* *} P<0.01,{ }^{* * *} P<0.001$.

Another important finding from our study was that TLR7 activation in B cells was critical for IgA1 and Gd-IgA1 production in patients with IgAN in vitro. The levels of IgA1 produced by TLR7/8-activated B cells in vitro were positively associated with serum IgA concentration in patients with IgAN, suggesting that the intensity of B cells responding to TLR7/8 ligand critically affects the production of IgA1 in IgAN. High levels of both circulating and local deposition of Gd-IgA1 have been shown to contribute to progressive renal injury (67-69). Thus, enhanced production of IgA1 and Gd-IgA1 from TLR7-expressing B cells could contribute to development and progression of IgAN. Patients with lupus exhibit high expression of TLR7 in PBMCs and B cells $(70,71)$. High TLR7 expression is related to human transitional B cell expansion and autoantibody production in patients with lupus (72). These findings, together with the data presented in our study, support the pathogenic role of TLR7 in boosting antibody synthesis in glomerulonephritis, including IgAN and lupus nephritis.

Mechanistically, we found that TLR7 was responsible for production of IgA1 and Gd-IgA1 as blockade of TLR7 but not TLR8 effectively inhibited production of IgA1 and Gd-IgA1 in activated PBMCs from patients with IgAN. The regulatory role for TLR7 was further confirmed by the fact that overexpression of TLR7 led to more GALNT2 proteins, whereas knockdown of TLR7 diminished 

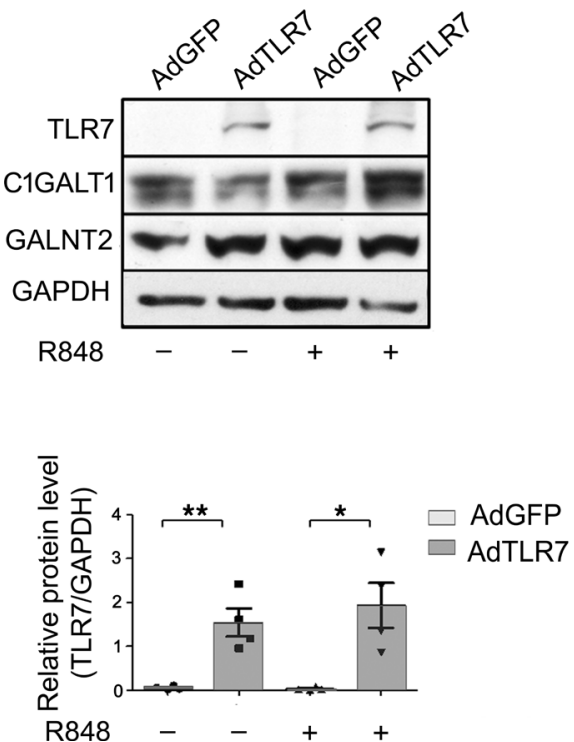
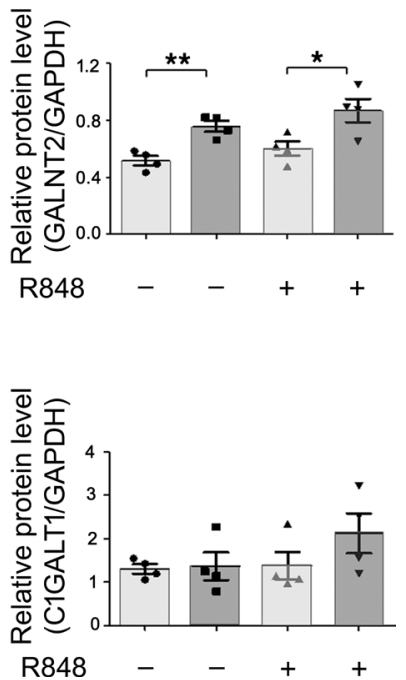

Figure 7. Overexpression of TLR7 protein resulted in increased expression of GALNT2 in HEK293T cells. Adenoviruses carrying coding sequences for TLR7 (AdTLR7) or GFP (AdGFP) were transfected into HEK293T cells, respectively, with the presence or absence of R848 $(1 \mu \mathrm{g} / \mathrm{mL})$. Cells were collected after 24 hours and analyzed by Western blot. Quantification of protein levels was presented $(n=4)$. Data were presented as mean \pm SEM and analyzed by 2-tailed unpaired Student's $t$ test. ${ }^{*} P<0.05,{ }^{* *} P<0.01$.

GALNT2 proteins. The hinge region of IgA1 contained more exposed GalNAc residues in patients with IgAN than in healthy individuals $(73,74)$, which may be explained by the relative insufficiency of Gal residues compared with GalNAc residues. Recently, Stewart et al. have shown that GALNT2 is a critical determinant of the number and pattern of $O$-glycans added to the hinge region of IgA1 molecules (75). Therefore, the ratio of C1GALT1/GALNT2 likely determines the $O$-glycan composition of IgA1 molecules, and our results support this hypothesis. We found that the ratio of C1GALT1/GALNT2 was significantly reduced in activated B cells from IgAN patients, and overexpression of GALNT2 led to significantly higher Gd-IgA1 production in B cell lines derived from IgAN patients. Recent evidence has shown that IL- 6 and APRIL synergistically affect the synthesis of Gd-IgA after TLR9 activation, and IL-6 could regulate the expression of the O-glycosyltransferases ST6GALNAC2 in vitro (20, 76). Based on these observations, we propose that TLR activation and downstream cytokines influence the aberrant expression of $O$-glycosyltransferases in IgAN.

Overall, TLR7 facilitates inflammatory responses in renal B cells and promotes Gd-IgA1 production in peripheral B cells of patients with IgAN. We hope these findings help to improve understanding of the role of TLR7 in B cells and provide a better insight of TLR7 as a therapeutic target for treating glomerulonephritis.

\section{Methods}

Supplemental Methods are available online with this article.

Subjects. Patients with biopsy evidence as primary IgAN, primary MCD, or primary MN were enrolled in the first affiliated hospital of Sun Yat-sen University (inclusion criteria seen in Supplemental Figure 3). Donors without CKDs were included as non-CKD controls. Healthy donors were negative for hematuria and proteinuria, and all met the criteria for normal serum creatinine content and hepatic function. Renal histopathology of patients with IgAN was classified according to the Oxford classifications with the 4 pathological variables: the mesangial hypercellularity $(\mathrm{M})$, the segmental glomerulosclerosis (S), the endocapillary hypercellularity (E), and the tubular atrophy/interstitial fibrosis (T). Global glomerulosclerosis was also scored according to the proportion of glomeruli that exhibited glomerulosclerosis (77).

Detection of target proteins in kidney biopsy specimens with immunofluorescence staining. Paraffin-embedded sections of biopsy specimens $(4 \mu \mathrm{M})$ were deparaffinized and rehydrated, followed by pressure cooking in citrate buffer $(0.01 \mathrm{M}, \mathrm{pH} 6.0)$. The sections were blocked in PBS containing $3 \%$ BSA and $0.3 \%$ Triton X-100 (MP Biomedicals); then, they were incubated with appropriate primary antibodies, 
A
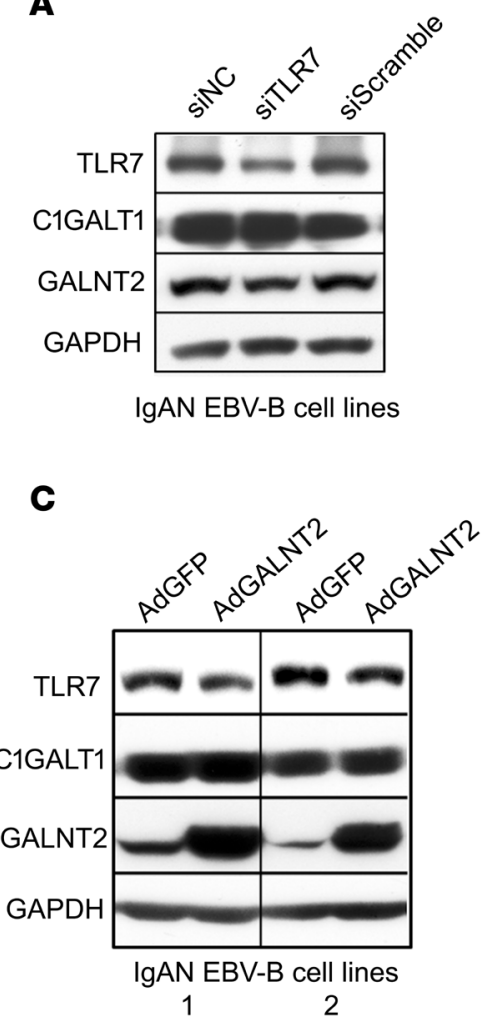

B

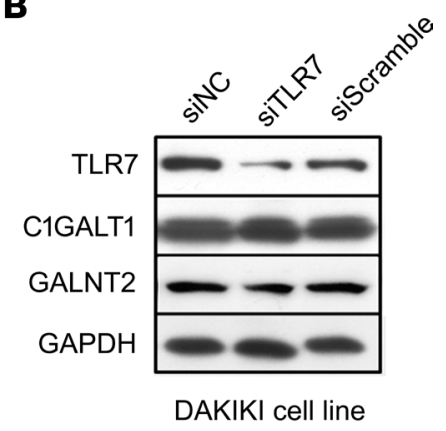

D
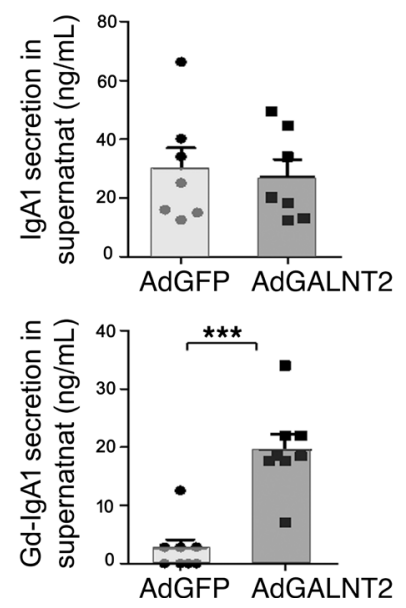

Figure 8. TLR7-GALNT2 axis affected galactose deficiency of IgA1 antibodies in B cells. (A and B) Synthetic siRNA targeting human TLR7 gene or control siRNA were electroporated into EBV-immortalized B cell lines derived from patients with IgAN (A) and DAKIKI B cell lines (B). siNC, negative control; siTLR7, siRNA for TLR7; siScramble, scramble siRNA control for TLR7. (C) Adenoviruses carrying coding sequences for GALNT2 (AdGALNT2) or GFP (AdGFP) gene were transfected into EBV-immortalized B cells derived from patients with IgAN. Cells were collected after 72 hours and analyzed by Western blot for target proteins. These experiments were repeated independently 3 times, and representative data was selected for display. (D) Culture supernatant was collected 6 days after transfection and analyzed by IgA1 and Gd-gA1 ELISA $(n=7)$. Data are presented as mean \pm SEM and analyzed by 2-tailed unpaired Student's $t$ test. ${ }^{* *} P<0.001$.

including rabbit anti-TLR7 antibody (Pierce, Thermo Fisher Scientific, PA1-28109), goat anti-CD19 antibody (Lifespan Bioscience, LSB-13077), mouse anti-CD20 antibody (Abcam, ab9475), and rabbit anti-TLR8 antibody (Lifespan Bioscience, LS-B1421). Secondary antibodies conjugated with different fluorescein were used to detect the primary antibodies. For frozen sections from renal biopsy specimens, samples were fixed and incubated with rabbit anti-IL-6 (Affinity Bioscience, DF6087), rabbit anti-IL-12 (Affinity Bioscience, AF5133), rabbit anti-Ki67 (Affinity Bioscience, AF0198), mouse antiTLR7 (R\&D Systems, MAB5875), mouse anti-IgA (Santa Cruz Biotechnology, sc271913), and goat anti-CD19 (Lifespan Bioscience, LSB-13077) antibodies, followed by secondary fluorescein-conjugated antibodies. Finally, nuclei were stained by DAPI, and slides were washed extensively with PBS before mounting. Digital images of the experimental glass slides were obtained with confocal laser scanning microscopy (Zeiss 880, Leica). Quantification of mean fluorescence intensity was performed with Zeiss Software Zen. At least 5 different fields from each slide were measured and averaged to obtain the arithmetic mean intensities of target proteins (original magnification, $\times 400$ ).

Preparation of PBMCS and gene expression analysis. PBMCs from venous blood were prepared by Ficoll-paque (GE Healthcare, 17-1440-02) density centrifugation. After centrifugation at $800 \mathrm{~g}$ at $20^{\circ} \mathrm{C}$ for 20 minutes, cells were washed 3 times with PBS containing 2 mM EDTA. The viability was above $95 \%$, as observed by eosin red staining. To isolate fresh CD $19^{+}$cells, PBMCs were labeled with CD19 microbeads (Miltenyi Biotec, 130-050-301) and isolated by magnetic separation. Total RNA of cells was extracted using TRIzol (Thermo Fisher Scientific, 15596026) reagent and treated by DNase I 
(Thermo Fisher Scientific, AM2235) digestion. A total of 200 ng of RNA from each sample was subjected to reverse transcription reaction for 30 -minute incubation at $37^{\circ} \mathrm{C}$ with a cDNA synthesis kit (TAKARA, RR037A). The cDNA nucleotide sequences of C1GALT1, COSMC, GALNT2, TLR7, $T L R 8$, and GAPDH genes were acquired from the National Center for Biotechnology Information's database (https://www.ncbi.nlm.nih.gov/). Primers for each gene were designed with Primer 3.0 software and confirmed with nonredundancy (Supplemental Figure 7). The system of real-time PCR reaction was prepared following instructions of a real-time PCR kit (TAKARA, RR420A), and reaction was carried out with an ABI7900 instrument (Applied Biosystems, Thermo Fisher Scientific). The data were analyzed with SDS2.4 software and Microsoft Excel software. The relative gene expression was calculated with the $2^{-\Delta \mathrm{Ct}}$ method, using GAPDH as internal control.

In vitro culture of PBMCs with TLR7/8 ligands and inhibitors. Plates for PBMCs culture were precoated with anti-IgM antibodies (Jackson ImmunoResearch, 109-005-129) at a concentration of $5 \mu \mathrm{g} / \mathrm{mL}$ in $\mathrm{PBS}$ at $4^{\circ} \mathrm{C}$ overnight. PBMCs from patients or controls were isolated and resuspended in complete 1640 medium (RPMI 1640 medium, 10\% FBS, $100 \mu \mathrm{g} / \mathrm{mL}$ of penicillin and streptomycin, $2 \mathrm{mM}$ L-glutamine, $100 \mathrm{mM}$ sodium pyruvate, $1 \times$ non-essential amino acid, $55 \mu \mathrm{M} \beta$-mercaptoethanol) (Gibco, Thermo Fisher Scientific). Cells were cultured at $37^{\circ} \mathrm{C}$ in a $5 \% \mathrm{CO}_{2}$ incubator at the density of $2.5 \times 10^{5}$ cells per well in 96-well plate. The cells were stimulated with TLR7/8 ligand-R848 (Enzo Life Sciences, ALX420-038-M005) for up to 12 days. After culture, cells were lysed for Western blot analysis, and culture supernatant was harvested for IgA1 ELISA and lectin binding assay. For blockade of TLR7 activation, a synthetic small compound (Zinc 4756232), reported to be a TLR7 inhibitor (78), was synthesized by Specs. TLR8-specific inhibitor CU-CTP8m (79) (CAS 125079-83-6) was synthesized by DC Chemicals. Loxoribine (InvivoGen, tlrl-lox), a selective TLR7 agonist, was also included in the culture system at a concentration of $0.5 \mathrm{mM}$ to induce the synthesis of $\operatorname{IgA} 1$ and Gd-IgA1.

Measurement of IgA1 and Gd-IgA1 in ELISA. For measurement of IgA1 content in culture supernatant, 96-well plates were precoated with IgA1 antibodies (Lifespan Bioscience, LS-C141768) at $4^{\circ} \mathrm{C}$ overnight. The plates were then blocked with PBS containing $1 \%$ BSA and $1 \%$ milk for $2-4$ hours at $37^{\circ} \mathrm{C}$. The wells were incubated with diluted culture supernatant or standard IgA1 protein (Abcam, ab91020) for 4 hours at $37^{\circ} \mathrm{C}$. Then, the IgA1 proteins sequentially binded to biotinylated anti-IgA1 antibody (Abcam, ab128739) and peroxidase-conjugated streptavidin (Thermo Fisher Scientific, 21130). Between each step, the plates were washed extensively with PBS-Tween. The color was developed with tetramethyl benzidine (TMB) and stopped by $2 \mathrm{~N} \mathrm{HCl}$. The optical density of each well was measured at $\mathrm{OD}_{450}$ by Spectrophotometer (SpectraMax M5, Molecular Devices).

Lectin binding assay was conducted according to ref. 80, with minor modification. Briefly, supernatant samples from PBMCs culture were ultracentrifuged at $1000 \mathrm{~g}$ at $4^{\circ} \mathrm{C}$ for 20 minutes and adjusted to a concentration of $1 \mu \mathrm{g} / \mathrm{mL}$. A total of $50 \mu \mathrm{L}$ of the concentrated supernatant was applied to each well of the high-binding plate precoated with anti-IgA antibody (DAKO, A026201-2). After overnight incubation and washing, neuraminidases (Roche, 11080725001) were incubated with samples at the concentration of $15 \mathrm{mIU} / \mathrm{mL}$ at $37^{\circ} \mathrm{C}$ for 3 hours in $100 \mathrm{mM}$ sodium citrate buffer (pH 5.0). Biotinylated HAA lectins (MilliporeSigma, L8764, SLBK5116V) were then applied at concentration of $1 \mu \mathrm{g} / \mathrm{mL}$ in $100 \mu \mathrm{L}$ PBS and incubated for 2 hours at room temperature. The lectin binding was detected with HRP-conjugated streptavidin for 1 hour at room temperature, followed by color development. In another plate, IgA1 binding was detected with biotinylated anti-IgA1 antibody instead of HAA lectin, followed by HRP-streptavidin binding as mentioned above. The results were expressed as HAA-binding absorbance adjusted for IgA1 binding absorbance to correct minor variations of IgA1 concentrations in different samples.

For supernatant collected from EBV-immortalized B cells, Gd-IgA1 ELISA kits (Immuno-Biological Laboratories, 27600) were used to measure the content of Gd-IgA1. Briefly, antibodies specific for Gd-IgA1 (KM55) (included in the ELISA kit) were precoated onto wells. Supernatant samples or standard of Gd-IgA1 were incubated in plate for 2 hours at $37^{\circ} \mathrm{C}$, followed by wash and incubation with HRP-conjugated anti-human IgA1 (included in the ELISA kit) antibody for 1 hour at $37^{\circ} \mathrm{C}$. Finally, color was developed and measured at $\mathrm{OD}_{450}$.

Detection of O-glycosyltransferases in stimulated PBMCs and B cells by Western blot. Proteins in target cells were extracted using protein lysis buffer (Beyotime, P0013) and separated in SDS-PAGE. The proteins were sequentially transferred to PVDF membrane; blocked with 5\% dry milk/PBS; incubated with primary antibodies for human TLR7 (Abcam, ab124928), IgA (DAKO, A026201-2), C1GALT1 (Abcam, ab57492), 
and GALNT2 (Thermo Fisher Scientific, PA5-21541); and finally incubated with secondary antibodies for chemiluminescence development.

To analyze expression of C1GALT1 and GALNT2 in B cells, PBMCs were stimulated with R848 for 9 days in vitro; then, the cells were labeled with CD19 antibody conjugated with APC (Thermo Fisher Scientific, 17-0199-42). Cells were sorted in a Moflo instrument (Beckman Coulter) and prepared for Western blot analysis as mentioned above.

Manipulating expression of TLR7 and GALNT2 in human cell lines. HEK293T cells (ATCC, CRL-11268) were cultured in DMEM high-glucose medium supplemented with $10 \%$ FBS. DAKIKI cell line (ATCC, TIB-206) was cultured in ATCC modified RPMI medium supplemented with 10\% FBS. EBV-immortalized $\mathrm{B}$ cells from patients with IgAN were generated as in ref. 81. Briefly, PBMCs from patients with IgAN were incubated with culture supernatant from B95-8 cells (ATCC, VR-1492) and cycloporin A (Solarbio, C8781) for about 4 weeks, and cell clusters with B cell phenotypes were formed (Supplemental Figure 4). EBV-immortalized B cells within 6 passages were used for experiments.

Adenoviruses harboring coding sequences for human GALNT2 and TLR7 gene were prepared by Vigene Biosciences and confirmed by DNA sequencing. Adenovirus particles containing TLR7, GALNT2, or GFP were respectively incubated with target cells at MOI of $1 \times 10^{3}$ to $1 \times 10^{4}: 1$. Protein samples were harvested and subjected to Western blot analysis.

To knock down expression of TLR7 gene, siRNA for TLR7 (sense, 5' GACCUUGGAUCUAAGUAAAUU 3'; antisense, 5' UUUACUUAGAUCCAAGGUCUU 3') was synthesized by GenePharma. Scramble siRNA (sense, 5' UUUAUAAAGAAGGGACUCCUU 3'; antisense, 5' GGAGUCCCUUCUUUAUAAAUU $3^{\prime}$ ) and negative control were synthesized as controls. A total of $100 \mathrm{pM}$ of siRNA was electroporated into B cell lines using Amaxa Cell Line Nucleofector Kit V (Lonza, VPA1003). Protein samples from cells were analyzed by Western blot analysis after 72 hours. Culture supernatant was collected for ELISA analysis after 6 days of culture.

Statistics. Graphs and statistical analyses were performed with GraphPad Prism 6.0 and SPSS (version 18.0) software. For normal distribution of quantitative variables, we used 2-tailed Student's $t$ tests and Pearson correlation coefficients. For nonnormally distributed variables, we analyzed with Mann-Whitney $U$ test and Spearman's correlation coefficients. One-way ANOVA followed by a post hoc test was used for multiple group comparisons. A 2-tailed $P<0.05$ was considered statistically significant. Data are shown as mean \pm SEM.

Study approval. All work contained in this publication was approved by the ethics review committee of the first affiliated hospital of Sun Yat-sen University, Guangzhou, China. All participants gave written informed consent in accordance with the ethics principles stated in the Declaration of Helsinki.

\section{Author contributions}

$\mathrm{XY}, \mathrm{NZ}$, and WC designed the study and wrote the manuscript. NZ, KX, HY, YD, and JF performed the experiments and analyzed the data. BW, NL, and JT prepared the blood samples and renal biopsy samples.

\section{Acknowledgments}

This project was supported financially by the National Natural Science Foundation of China (31200664), Chinese National Key Technology R and D Program, Ministry of Science and Technology (2017YFC0907601, 2017YFC0907602, 2017YFC0907603, 2016YFC0906100, 2016YFC0906101); National Natural Science Foundation of China (81970599); Guangzhou Municipal Program of Science and Technology (201804020049); Key Laboratory of National Health Commission; and Key Laboratory of Nephrology, Guangzhou, Guangdong Province, China (2002B60118, 2017B030314019). We appreciated the help of Zhiqiang Huang from University of Alabama (Birmingham, Alabama, USA), Huiyao Lan from the Chinese University of Hong Kong (Hong Kong, China), and Karen Molyneux from University of Leicester (Leicester, United Kingdom) for their technical support and helpful advice in manuscript preparation.

Address correspondence to: Xueqing Yu, Wei Chen, or Nuoyan Zheng, Department of Nephrology, The First Affiliated Hospital of Sun Yat-sen University, 58th Zhongshan Er Road, Yuexiu District, Guangzhou, 510000, China. Phone: 8620.83827812.21168; Email: yuxq@mail.sysu.edu.cn (XY), vvchen66@qq.com (WC), or zhnuoy@mail.sysu.edu.cn (NZ). 
1. Barratt J, Feehally J, Smith AC. Pathogenesis of IgA nephropathy. Semin Nephrol. 2004;24(3):197-217.

2. Barratt J, Feehally J. IgA nephropathy. J Am Soc Nephrol. 2005;16(7):2088-2097.

3. Lai KN, et al. IgA nephropathy. Nat Rev Dis Primers. 2016;2:16001.

4. Knoppova B, et al. The origin and activities of IgA1-containing immune complexes in IgA nephropathy. Front Immunol. 2016;7:117.

5. Kiryluk K, Novak J. The genetics and immunobiology of IgA nephropathy. J Clin Invest. 2014;124(6):2325-2332.

6. Suzuki H, et al. Aberrantly glycosylated IgA1 in IgA nephropathy patients is recognized by IgG antibodies with restricted heterogeneity. J Clin Invest. 2009;119(6):1668-1677.

7. Floege J, Moura IC, Daha MR. New insights into the pathogenesis of IgA nephropathy. Semin Immunopathol. 2014;36(4):431-442.

8. Suzuki Y, et al. Serum levels of galactose-deficient immunoglobulin (Ig) A1 and related immune complex are associated with disease activity of IgA nephropathy. Clin Exp Nephrol. 2014;18(5):770-777.

9. Zhao N, et al. The level of galactose-deficient IgA1 in the sera of patients with IgA nephropathy is associated with disease progression. Kidney Int. 2012;82(7):790-796.

10. Berthoux F, et al. Autoantibodies targeting galactose-deficient IgA1 associate with progression of IgA nephropathy. $J A m$ Soc Nephrol. 2012;23(9):1579-1587.

11. Yanagawa $\mathrm{H}$, et al. A panel of serum biomarkers differentiates IgA nephropathy from other renal diseases. PLoS One. 2014;9(5):e98081.

12. Rauen T, Floege J. Inflammation in IgA nephropathy. Pediatr Nephrol. 2017;32(12):2215-2224.

13. Floege J, Feehally J. The mucosa-kidney axis in IgA nephropathy. Nat Rev Nephrol. 2016;12(3):147-156.

14. Suzuki H, et al. IgA1-secreting cell lines from patients with IgA nephropathy produce aberrantly glycosylated IgA1. J Clin Invest. 2008;118(2):629-639.

15. Smith AC, Molyneux K, Feehally J, Barratt J. O-glycosylation of serum IgA1 antibodies against mucosal and systemic antigens in IgA nephropathy. J Am Soc Nephrol. 2006;17(12):3520-3528.

16. Novak J, Barratt J, Julian BA, Renfrow MB. Aberrant glycosylation of the IgA1 molecule in IgA nephropathy. Semin Nephrol. 2018;38(5):461-476.

17. Iwasaki H, et al. Initiation of O-glycan synthesis in IgA1 hinge region is determined by a single enzyme, UDP-N-acetyl-alpha-D-galactosamine:polypeptide N-acetylgalactosaminyltransferase 2. J Biol Chem. 2003;278(8):5613-5621.

18. Ju T, Brewer K, D'Souza A, Cummings RD, Canfield WM. Cloning and expression of human core 1 beta1,3-galactosyltransferase. J Biol Chem. 2002;277(1):178-186.

19. Ju T, Cummings RD. A unique molecular chaperone Cosmc required for activity of the mammalian core 1 beta 3-galactosyltransferase. Proc Natl Acad Sci U S A. 2002;99(26):16613-16618.

20. Suzuki H, et al. Cytokines alter IgA1 O-glycosylation by dysregulating C1GalT1 and ST6GalNAc-II enzymes. J Biol Chem. 2014;289(8):5330-5339.

21. Qin W, et al. Peripheral B lymphocyte beta1,3-galactosyltransferase and chaperone expression in immunoglobulin A nephropathy. J Intern Med. 2005;258(5):467-477.

22. Yamada K, et al. Down-regulation of core 1 beta1,3-galactosyltransferase and Cosmc by Th2 cytokine alters O-glycosylation of IgA1. Nephrol Dial Transplant. 2010;25(12):3890-3897.

23. Inoue $\mathrm{T}$, et al. Differential expression of glycogenes in tonsillar B lymphocytes in association with proteinuria and renal dysfunction in IgA nephropathy. Clin Immunol. 2010;136(3):447-455.

24. Heller F, et al. The contribution of B cells to renal interstitial inflammation. Am J Pathol. 2007;170(2):457-468.

25. Chang A, et al. In situ B cell-mediated immune responses and tubulointerstitial inflammation in human lupus nephritis. JImmunol. 2011;186(3):1849-1860.

26. Shen Y, et al. Association of intrarenal B-cell infiltrates with clinical outcome in lupus nephritis: a study of 192 cases. Clin Dev Immunol. 2012;2012:967584

27. Segerer S, Schlöndorff D. B cells and tertiary lymphoid organs in renal inflammation. Kidney Int. 2008;73(5):533-537.

28. Herlands RA, Christensen SR, Sweet RA, Hershberg U, Shlomchik MJ. T cell-independent and Toll-like receptor-dependent antigen-driven activation of autoreactive B cells. Immunity. 2008;29(2):249-260.

29. Meiler F, Klunker S, Zimmermann M, Akdis CA, Akdis M. Distinct regulation of IgE, IgG4 and IgA by T regulatory cells and Toll-like receptors. Allergy. 2008;63(11):1455-1463.

30. Takemura S, Klimiuk PA, Braun A, Goronzy JJ, Weyand CM. T cell activation in rheumatoid synovium is B cell dependent. J Immunol. 2001;167(8):4710-4718.

31. Muto M, et al. Toll-like receptor 9 stimulation induces aberrant expression of a proliferation-inducing ligand by tonsillar germinal center B cells in IgA nephropathy. J Am Soc Nephrol. 2017;28(4):1227-1238.

32. Nakata J, et al. Changes in nephritogenic serum galactose-deficient IgA1 in IgA nephropathy following tonsillectomy and steroid therapy. PLoS One. 2014;9(2):e89707.

33. Sato D, et al. Tonsillar TLR9 expression and efficacy of tonsillectomy with steroid pulse therapy in IgA nephropathy patients. Nephrol Dial Transplant. 2012;27(3):1090-1097.

34. Kajiyama T, Suzuki Y, Kihara M, Suzuki H, Horikoshi S, Tomino Y. Different pathological roles of Toll-like receptor 9 on mucosal B cells and dendritic cells in murine IgA nephropathy. Clin Dev Immunol. 2011;2011:819646.

35. Makita Y, et al. TLR9 activation induces aberrant IgA glycosylation via APRIL- and IL-6-mediated pathways in IgA nephropathy. Kidney Int. 2020;97(2):340-349.

36. Coppo R, et al. Toll-like receptor 4 expression is increased in circulating mononuclear cells of patients with immunoglobulin A nephropathy. Clin Exp Immunol. 2010;159(1):73-81.

37. Donadio ME, et al. Toll-like receptors, immunoproteasome and regulatory T cells in children with Henoch-Schönlein purpura and primary IgA nephropathy. Pediatr Nephrol. 2014;29(9):1545-1551.

38. Heil F, et al. Species-specific recognition of single-stranded RNA via Toll-like receptor 7 and 8. Science. 2004;303(5663):1526-1529.

39. Diebold SS. Recognition of viral single-stranded RNA by Toll-like receptors. Adv Drug Deliv Rev. 2008;60(7):813-823.

40. Hornung V, et al. Quantitative expression of Toll-like receptor 1-10 mRNA in cellular subsets of human peripheral blood 
mononuclear cells and sensitivity to CpG oligodeoxynucleotides. J Immunol. 2002;168(9):4531-4537.

41. Gorden KB, et al. Synthetic TLR agonists reveal functional differences between human TLR7 and TLR8. J Immunol. 2005; 174(3):1259-1268.

42. Saito A, et al. Different expression patterns of Toll-like receptor mRNAs in blood mononuclear cells of IgA nephropathy and IgA vasculitis with nephritis. Tohoku J Exp Med. 2016;240(3):199-208.

43. Zheng N, et al. Expression profile of BAFF in peripheral blood from patients of IgA nephropathy: correlation with clinical features and Streptococcus pyogenes infection. Mol Med Rep. 2017;15(4):1925-1935.

44. Pei G, et al. Renal interstitial infiltration and tertiary lymphoid organ neogenesis in IgA nephropathy. Clin J Am Soc Nephrol. 2014;9(2):255-264.

45. Ciferska H, et al. Does the renal expression of Toll-like receptors play a role in patients with IgA nephropathy? J Nephrol. 2020;33(2):307-316

46. Glaum MC, et al. Toll-like receptor 7-induced naive human B-cell differentiation and immunoglobulin production. J Allergy Clin Immunol. 2009;123(1):224-230.e4.

47. Bishop GA, Ramirez LM, Baccam M, Busch LK, Pederson LK, Tomai MA. The immune response modifier resiquimod mimics CD40-induced B cell activation. Cell Immunol. 2001;208(1):9-17.

48. Lee J, et al. Molecular basis for the immunostimulatory activity of guanine nucleoside analogs: activation of Toll-like receptor 7. Proc Natl Acad Sci U S A. 2003;100(11):6646-6651.

49. Bekeredjian-Ding I, Jego G. Toll-like receptors--sentries in the B-cell response. Immunology. 2009;128(3):311-323.

50. Ballardie FW, Gordon MT, Sharpe PT, Darvill AM, Cheng H. Intrarenal cytokine mRNA expression and location in normal and IgA nephropathy tissue: TGF alpha, TGF beta, IGF 1, IL-4 and IL-6. Nephrol Dial Transplant. 1994;9(11):1545-1552.

51. Taniguchi Y, et al. Interleukin-6 localization and the prognosis of IgA nephropathy. Nephron. 1999;81(1):94-98.

52. Taniguchi Y, Yorioka N, Tanji C, Asakimori Y, Yamakido M. Immunohistochemical study of interleukin 12 in patients with IgA nephropathy. Nephron. 2000;86(3):370-371.

53. Yano N, Endoh M, Nomoto Y, Sakai H, Fadden K, Rifai A. Phenotypic characterization of cytokine expression in patients with IgA nephropathy. J Clin Immunol. 1997;17(5):396-403.

54. Su H, Lei CT, Zhang C. Interleukin-6 signaling pathway and its role in kidney disease: an update. Front Immunol. 2017;8:405.

55. Kim DI, Park SH. Sequential signaling cascade of IL-6 and PGC-1 $\alpha$ is involved in high glucose-induced podocyte loss and growth arrest. Biochem Biophys Res Commun. 2013;435(4):702-707.

56. Ruef C, et al. Interleukin 6 is an autocrine growth factor for mesangial cells. Kidney Int. 1990;38(2):249-257.

57. Xiao Y, Yang N, Zhang Q, Wang Y, Yang S, Liu Z. Pentraxin 3 inhibits acute renal injury-induced interstitial fibrosis through suppression of IL-6/Stat3 pathway. Inflammation. 2014;37(5):1895-1901.

58. Schrader LI, Kinzenbaw DA, Johnson AW, Faraci FM, Didion SP. IL-6 deficiency protects against angiotensin II induced endothelial dysfunction and hypertrophy. Arterioscler Thromb Vasc Biol. 2007;27(12):2576-2581.

59. Schwarting A, Tesch G, Kinoshita K, Maron R, Weiner HL, Kelley VR. IL-12 drives IFN-gamma-dependent autoimmune kidney disease in MRL-Fas(lpr) mice. J Immunol. 1999;163(12):6884-6891.

60. Kitching AR, et al. IL-12p40 and IL-18 in crescentic glomerulonephritis: IL-12p40 is the key Th1-defining cytokine chain, whereas IL-18 promotes local inflammation and leukocyte recruitment. J Am Soc Nephrol. 2005;16(7):2023-2033.

61. Pawar RD, et al. Toll-like receptor-7 modulates immune complex glomerulonephritis. J Am Soc Nephrol. 2006;17(1):141-149.

62. Patole PS, et al. Coactivation of Toll-like receptor-3 and -7 in immune complex glomerulonephritis. J Autoimmun. 2007;29(1):52-59.

63. Feng Y, Yang S, Ma Y, Bai XY, Chen X. Role of Toll-like receptors in diabetic renal lesions in a miniature pig model. Sci Adv. 2015;1(5):e1400183.

64. Yayi H, Yeda X, Huaxin W, Yang W, Qian S, Zhongyuan X. Toll-like receptor 7 involves the injury in acute kidney ischemia/ reperfusion of STZ-induced diabetic rats. Acta Cir Bras. 2016;31(7):448-455.

65. Celhar T, et al. TLR7 Protein expression in mild and severe lupus-prone models is regulated in a leukocyte, genetic, and IRAK4 dependent manner. Front Immunol. 2019;10:1546.

66. Conti F, et al. Kidney expression of Toll like receptors in lupus nephritis: quantification and clinicopathological correlations Mediators Inflamm. 2016;2016:7697592.

67. Ebefors K, et al. Mesangial cells from patients with IgA nephropathy have increased susceptibility to galactose-deficient IgA1. BMC Nephrol. 2016;17:40.

68. Amore A, Cirina P, Conti G, Brusa P, Peruzzi L, Coppo R. Glycosylation of circulating IgA in patients with IgA nephropathy modulates proliferation and apoptosis of mesangial cells. J Am Soc Nephrol. 2001;12(9):1862-1871.

69. Nguyen C, et al. Higher serum galactose-deficient immunoglobulin A1 concentration is associated with stronger mesangial cellular inflammatory response and more severe histologic findings in immunoglobulin A nephropathy. Clin Kidney J. 2019;12(2):232-238

70. Lyn-Cook BD, et al. Increased expression of Toll-like receptors (TLRs) 7 and 9 and other cytokines in systemic lupus erythematosus (SLE) patients: ethnic differences and potential new targets for therapeutic drugs. Mol Immunol. 2014;61(1):38-43.

71. Klonowska-Szymczyk A, Wolska A, Robak T, Cebula-Obrzut B, Smolewski P, Robak E. Expression of Toll-like receptors 3, 7, and 9 in peripheral blood mononuclear cells from patients with systemic lupus erythematosus. Mediators Inflamm. 2014;2014:381418.

72. Wang T, et al. High TLR7 expression drives the expansion of CD $19^{+} \mathrm{CD} 24^{\mathrm{hi}} \mathrm{CD} 38^{\text {hi }}$ transitional B cells and autoantibody production in SLE patients. Front Immunol. 2019;10:1243.

73. Gastaldi D, et al. Mass spectrometry analysis of IgA1 hinge region in patients with IgA nephropathy. J Nephrol. 2007;20(6):689-695.

74. Ohyama Y, et al. Analysis of O-glycoforms of the IgA1 hinge region by sequential deglycosylation. Sci Rep. 2020;10(1):671.

75. Stewart TJ, et al. IgA1 hinge-region clustered glycan fidelity is established early during semi-ordered glycosylation by GalNAc-T2. Glycobiology. 2019;29(7):543-556.

76. Makita Y, et al. TLR9 activation induces aberrant IgA glycosylation via APRIL- and IL-6-mediated pathways in IgA nephropathy. Kidney Int. 2020;97(2):340-349.

77. Kataoka H, et al. Overweight and obesity accelerate the progression of IgA nephropathy: prognostic utility of a combination of 
BMI and histopathological parameters. Clin Exp Nephrol. 2012;16(5):706-712.

78. Svajger U, Horvat Z, Knez D, Rozman P, Turk S, Gobec S. New antagonists of Toll-like receptor 7 discovered through 3D ligand-based virtual screening. Med Chem Res. 2015;24(1):362-371.

79. Zhang S, et al. Small-molecule inhibition of TLR8 through stabilization of its resting state. Nat Chem Biol. 2018;14(1):58-64.

80. Smith AC, de Wolff JF, Molyneux K, Feehally J, Barratt J. O-glycosylation of serum IgD in IgA nephropathy. J Am Soc Nephrol. 2006;17(4):1192-1199.

81. Steinitz M, Klein E. Human monoclonal antibodies produced by immortalization with Epstein-Barr virus. Immunol Today $1981 ; 2(2): 38-39$. 